\title{
VANISHING OF ODD DIMENSIONAL INTERSECTION COHOMOLOGY II
}

\author{
Michel Brion AND Roy JoshuA
}

\begin{abstract}
For a variety where a connected linear algebraic group acts with only finitely many orbits, each of which admits an attractive slice, we show that the stratification by orbits is perfect for equivariant intersection cohomology with respect to any equivariant local system. This applies to provide a relationship between the vanishing of the odd dimensional intersection cohomology sheaves and of the odd dimensional global intersection cohomology groups. For example, we show that odd dimensional intersection cohomology sheaves and global intersection cohomology groups vanish for all complex spherical varieties.
\end{abstract}

\section{Introduction.}

This paper should be viewed as a continuation of the paper [J-1] by the second author. There equivariant intersection cohomology was applied to provide a geometric proof of the vanishing of odd dimensional local and global intersection cohomology for Schubert varieties and also certain other varieties. We briefly recall this situation as follows. Let $X$ denote a projective variety provided with the action of a torus $T$ and such that the odd dimensional middle intersection cohomology groups of $X$ are trivial. We showed that now the odd dimensional middle intersection cohomology sheaves of $X$ also vanish provided the following criteria are satisfied: there exists a $T$-stable decomposition of $X$ into locally closed smooth strata so that (i) each stratum has a $T$-fixed point and (ii) the middle intersection cohomology sheaves are locally constant on each stratum.

Unfortunately condition (i) is not satisfied in many situations: for example complete toric or spherical varieties and orbit closures of symmetric subgroups in flag manifolds. In the latter case, the vanishing of all odd dimensional intersection cohomology sheaves was obtained by Lusztig and Vogan, see [L-V]. The proof is rather involved, using a generalization of the Kazhdan-Lusztig theory and also representation theory of real reductive groups. Another proof, using an analysis of the orbit structure, has been obtained recently by Mars and Springer; see [M-S].

One of the original motivations for this paper was to give a general geometric explanation of this vanishing phenomenon using equivariant intersection cohomology (see [Bryl] and [J-1]) which is a variant of intersection cohomology that incorporates the group action into its very definition. One of our main results (Theorem 2) is that the the stratification by orbits is perfect for equivariant intersection cohomology of certain varieties. Using this as a tool, we obtain several general results relating the vanishing of the global odd dimensional intersection cohomology with the vanishing of the odd dimensional intersection cohomology sheaves with respect to equivariant local systems. For example we show the vanishing of the odd dimensional intersection cohomology sheaves with respect to the constant local system (up to taking certain invariants) for the orbit closures of symmetric subgroups on flag manifolds (Theorem 5). Using the local structure of spherical varieties, we also show that the odd dimensional intersection cohomology sheaves vanish for these varieties and all local systems (Theorem 4).

All the results in this paper extend to arbitrary characteristics. However, the proofs of Theorems 1 and 4 are much simpler over the field of complex numbers, whereas given Theorem 1, the proofs of Theorem 2, Corollary 3 and Theorem 5 adapt to positive characteristics with minor modifications. Theorem 1 is extended to positive characteristics in the accompanying paper [J-4] by the second author; (modulo a technical condition) Theorem 4 is extended to positive characteristics in a forthcoming paper [J-5].

The second author thanks the Max Planck Institut für Mathematik, Bonn for support 
Most of our results are stated explicitly only in characteristic 0 ; however we provide all the definitions in arbitrary characteristics so that the statements make sense in full generality.

We thank the referee for several valuable suggestions.

\section{Notation and conventions}

(0.1) Throughout this paper, $k$ will denote an algebraically closed field of arbitrary characteristic $p \geq 0$. We denote by $G$ a linear algebraic group, and by $G^{0}$ the connected component of the identity in $G$. A separated reduced scheme $X$ of finite type over $k$ will be called a variety; observe that varieties need not be irreducible. If $X$ is provided with an algebraic action of $G$, we will say that $X$ is a $G$-variety.

A $G$-variety will be called locally linear if it is a union of $G$-stable open subvarieties, each of them admitting a $G$-equivariant locally closed embedding into the projectivization of a $G$-module. For example, any normal $G$-variety is locally linear (this follows from [Su] Theorem I and Lemma 8 if $G$ is connected, and it is shown in [J-3] (1.9) that the hypothesis that $G$ be connected may be dropped.) We will only consider varieties which are locally linear and equidimensional.

(0.2) Consider a $G$-variety $X$ and a point $x \in X$; let $G x$ be its $G$-orbit and $G_{x}$ its isotropy group. A slice to $G x$ at $x$ is a locally closed subvariety $\mathcal{S}$ of $X$ containing $x$ and satisfying the following two conditions:

(i) There exists a maximal torus $T_{x}$ of $G_{x}$ such that $\mathcal{S}$ is stable under $T_{x}$.

(ii) The map $G \times \mathcal{S} \rightarrow X$ sending $(g, x)$ to $g x$ is smooth at $(e, x)$, and the dimension of $\mathcal{S}$ is the codimension of $G x$ in $X$.

Note that $\mathcal{S}$ exists if and only if the orbit map $G \rightarrow G x$ is separable. In particular, $\mathcal{S}$ always exists in characteristic 0 ; then it may be chosen stable under a maximal reductive subgroup of $G_{x}$. Moreover, by shrinking $\mathcal{S}$ if necessary, we may assume that the map $G \times \mathcal{S} \rightarrow X$ is smooth everywhere and that $\mathcal{S}$ is affine.

(0.3) Let $T$ denote a torus acting on a variety $X$ with a fixed point $x$. We say that $x$ is attractive if there exists a one parameter subgroup $\lambda: \mathbb{G}_{m} \rightarrow T$ such that, for all $y$ in a Zariski neighborhood of $x$, we have $\lim _{t \rightarrow 0} \lambda(t) y=x$. Equivalently, all weights of $T$ acting on the Zariski tangent space at $x$ are contained in an open half-space. In the situation of (0.2), we say that $\mathcal{S}$ is an attractive slice, if $x$ is an attractive fixed point for the action of $T_{x}$ on $\mathcal{S}$. (See Appendix (A.1) for further details on attractive fixed points.)

(0.4) Let $X$ be a variety. We denote by $H^{*}(X)$ the cohomology ring of $X$ with rational coefficients in characteristic 0 (the $l$-adic cohomology of $X$ in positive characteristics). If $\mathcal{L}$ is a local system (see Section 1$)$ on a smooth open subvariety of $X$, then $I H^{*}(X ; \mathcal{L})$ will denote the corresponding intersection cohomology for the middle perversity. If moreover $\mathcal{L}$ is constant, we simply write $I H^{*}(X)$ for $I H^{*}(X ; \mathcal{L})$.

For a $G$-equivariant local system (see Section 1$)$, we will denote by $H_{G}^{*}(X ; \mathcal{L})\left(I H_{G}^{*}(X ; \mathcal{L})\right)$ the corresponding equivariant cohomology (the equivariant intersection cohomology for the middle perversity, respectively); these are discussed in Section 1. Both $H_{G}^{*}(X)$ and $I H_{G}^{*}(X ; \mathcal{L})$ are modules over $H^{*}(B G)$, the equivariant cohomology ring of the point.

For any integer $n$, we denote by $\mathcal{H}^{n}(I C(X ; \mathcal{L}))$ the $n$-th cohomology sheaf of the middle intersection cohomology complex on $X$. The stalk of the sheaf $\mathcal{H}^{n}(I C(X ; \mathcal{L}))$ at a point $x$ will be denoted $\mathcal{H}^{n}(I C(X ; \mathcal{L}))_{x}$, while the local intersection cohomology with support in $x$ will be denoted $I H_{x}^{n}(X ; \mathcal{L})$. They are related as follows: $I H_{x}^{n}(X ; \mathcal{L})$ is the dual space of $\mathcal{H}^{n}\left(I C\left(X ; \mathcal{L}^{\vee}\right)\right)_{x}[2 d]$ where $\mathcal{L}^{\vee}$ denotes the dual of $\mathcal{L}$ and $d$ denotes the dimension of $X$.

(0.5) In characteristic $p>0$, we will assume all the varieties we consider are obtained by base extensions from varieties defined over some finite extension of $\mathbb{F}_{p}$.

(0.6) Throughout the paper we adopt the cohomology notation for perverse sheaves as in [J-1]. i.e. a complex of sheaves $K$ on a variety $X$ of dimension $d$ is perverse if the dimensions of the supports of the sheaves $\mathcal{H}^{n}(K)$ and $\mathcal{H}^{n}(D(K)[-2 d]$ ) are $\leq d-n$ for all $n$. (In [B-B-D] a complex $K$ is defined to be perverse if the dimensions of the supports of the sheaves $\mathcal{H}^{n}(K)$ and $\mathcal{H}^{n}(D(K))$ are $\leq-n$ for all $n$.) 
Now we begin with the following basic result that will be used repeatedly. (See section 1 for the notations. The equivariant derived category $D_{b}^{G}(X)$ is defined in (1.2.1) and (1.3.2) while equivariant hypercohomology $\mathbb{H}_{G}^{*}$ is defined in (1.3.6).)

Theorem 1. Let $H$ be a closed normal subgroup of $G$ such that the quotient $\bar{G}=G / H$ is finite. Let $X$ be a $G$-variety and let $K \in D_{b}^{G}(X)$.

(i) Identifying $K$ with its restriction to $D_{b}^{H}(X)$, there exists an action of $\bar{G}$ on $\mathbb{H}_{H}^{*}(X ; K)$ that is natural in $K$.

(ii) Moreover one has an identification $\mathbb{H}_{G}^{*}(X ; K) \cong \mathbb{H}_{H}^{*}(X ; K)^{\bar{G}}$.

(iii) In case $H=G^{0}$, one has an action of $\bar{G}$ on $\mathbb{H}_{G^{0}}^{*}(X ; K)$ that is natural in $K$. If moreover $G=G^{0} \times F$ for some finite group $F \cong G / G^{0}, F$ acts trivially on $X$ and $K$ is a $G$-equivariant sheaf on $X$, then $K^{F}$ is a $G^{0}$-equivariant sheaf and one has the isomorphisms

$$
H_{G}^{*}(X ; K) \cong H_{G^{0}}^{*}(X ; K)^{F} \cong H_{G^{0}}^{*}\left(X ; K^{F}\right) \cong H^{*}\left(B G^{0}\right) \otimes H^{*}\left(X ; K^{F}\right) .
$$

(iv) In case $\mathbb{H}_{H}^{*}(X ; K)$ is a free module over $H^{*}(B H)$, one has the identification

$$
\mathbb{H}_{G}^{*}(X ; D(K)) \cong\left(H_{o m}^{*}(B H)\left(\mathbb{H}_{H}^{*}(X ; K), H^{*}(B H)\right)\right)^{\bar{G}} .
$$

where $D(K)$ denotes the Verdier dual of $K$.

The proof of this theorem is considerably simpler in characteristic 0 where one has a fibration $B H \rightarrow$ $B G \rightarrow B \bar{G}$. The extension to positive characteristics requires the use of new techniques and is discussed in detail in the accompanying paper [J-4].

The following is our first main result.

Theorem 2. Let $X$ denote a $G$-variety containing only finitely many orbits, each of which admits an attractive slice. Let $\mathcal{L}$ denote a $G$-equivariant local system on the union of all open orbits in case $G$ is connected and a constant local system in case $G$ is not connected. Then the following hold.

(i) The $H^{*}(B G)$-module $I H_{G}^{*}(X ; \mathcal{L})$ admits a filtration with subquotients $I H_{\mathcal{O}, G}^{*}(X ; \mathcal{L})$ where $\mathcal{O}$ runs through the $G$-orbits in $X$ and $I H_{\mathcal{O}, G}^{*}(X ; \mathcal{L})$ denotes the equivariant intersection cohomology with supports in $\mathcal{O}$.

(ii) For $\mathcal{O}=G x$, the group of components $G_{x} / G_{x}^{0}$ acts on $H^{*}\left(B G_{x}^{0}\right)$ and on $I H_{x}^{*}(X ; \mathcal{L})$, and one has an isomorphism:

$$
I H_{\mathcal{O}, G}^{*}(X ; \mathcal{L}) \cong\left(H^{*+2 \operatorname{dim}(\mathcal{O})}\left(B G_{x}^{0}\right) \otimes I H_{x}^{*}(X ; \mathcal{L})\right)^{G_{x} / G_{x}^{0}}
$$

One may interpret statement (i) as saying that the stratification by orbits is perfect for equivariant intersection cohomology provided the hypotheses in Theorem 2 are satisfied. (This is analogous to a result of Kirwan: the stratification by "instability type" of any projective nonsingular G-variety is perfect for equivariant cohomology, see $[\mathrm{K}]$.)

Corollary 3. (i) Assume in addition to the hypotheses of Theorem 2 that $I H^{n}(X ; \mathcal{L})=0$ for all odd $n$. Then $\left(H^{*}\left(B G_{x}^{0}\right) \otimes I H_{x}^{*}(X ; \mathcal{L})\right)^{G_{x} / G_{x}^{0}}$ vanishes in all odd degrees and for all $x \in X$.

(ii) If, in addition, $G_{x}$ is connected, then $I H_{x}^{n}(X ; \mathcal{L})$ vanishes for all odd $n$.

(iii) Suppose in addition to the hypotheses in (i) that $G_{x}$ is the semi-direct product of a connected solvable group and a finite group $F_{x}$. Then $F_{x}$ acts on $I H_{x}^{*}(X ; \mathcal{L})$ and $\left(I H_{x}^{*}(X ; \mathcal{L})\right)^{F_{x}}$ vanishes in all odd degrees.

Observe that the hypotheses of (i) and (ii) are satisfied by all toric varieties (in fact, all their isotropy groups are connected). Therefore the above corollary establishes the vanishing of the odd dimensional intersection cohomology sheaves for toric varieties. (See also [B-B-F-K].) 
The following are the remaining results of the paper.

Theorem 4. Assume the ground field $k$ is of characteristic 0 . Let $G$ denote a connected reductive group and let $X$ denote a spherical $G$-variety. Let $\mathcal{L}$ denote a $G$-equivariant local system on the open $G$-orbit in $X$. Then $I H_{x}^{n}(X ; \mathcal{L})=0$ for all odd $n$ and all $x \in X$.

For the next statement, we assume that $p \neq 2$. Let $G$ denote a connected reductive group and let $\theta$ be an automorphism of order two of the algebraic group $G$. Let $K$ denote the fixed point subgroup of $\theta$ and let $B$ denote a Borel subgroup of $G$. Then $K$ acts on the flag manifold $G / B$ with finitely many orbits each of which admits an attractive slice by [M-S]. Our geometric methods yield the following theorem in Section 4.

Theorem 5. Let $X$ denote the closure of a $K$-orbit on $G / B$. Then $I H^{n}(X)$ and $\left(I H_{x}^{n}(X)\right)^{K_{x} / K_{x}^{0}}$ vanish for all odd $n$ and all $x \in X$.

(In fact, $I H_{x}^{n}(X ; \mathcal{L})$ vanishes for all $K$-equivariant local systems $\mathcal{L}$ on $X$ and for all odd $n$, see [L-V] and also [M-S].)

The organization of the paper is as follows. We begin with a brief review of equivariant derived categories and equivariant intersection cohomology in Section 1. (Some of the material here is available in the literature: but this is often stated only in characteristic 0 and for constant local systems, and the extension to positive characteristics is not straightforward.) We conclude this section with a technique whereby we are able to reduce consideration of local systems to the constant system, at least for the action of connected groups. Section 2 is devoted to the proof of Theorem 1. The main result of Section 3 is Theorem 2. Corollary 3 is also established there. In Section 4 we prove Theorems 4 and 5 . We discuss some details on actions of the multiplicative group in an Appendix.

\section{Equivariant intersection cohomology and equivariant derived categories}

We begin with the following definition of equivariant derived categories that is valid in characteristic 0 only.

(1.1.1) We let $E G$ denote the infinite join of $G$ with itself, otherwise called the Milnor construction. This is a contractible space on which $G$ acts freely and is functorial in $G$. Now $B G$ will denote the quotient $E G / G$. If $X$ is a $G$-variety, we let $E G \times X=(E G \times X) / G$ where we identify $(p, x)$ with $\left(p g^{-1}, g x\right)$. Now we obtain a fibration $X \rightarrow \underset{G}{E} \underset{G}{\times} \rightarrow B G$.

(1.1.2) What will be of crucial importance in the proof of Theorem 1 is the following observation. Let $\phi: G \rightarrow \bar{G}$ denote a surjective homomorphism of algebraic groups with kernel $H$. Now one obtains an induced locally trivial fibration $B \phi: B G \rightarrow B \bar{G}$ with fiber $B H$. This follows readily from the definition of Milnor construction. Since the existence of such a fibration is important for us, we will adopt this definition of EG and BG in characteristic 0, throughout the paper. (In positive characteristics, such a fibration does not exist and calls for the use of subtler techniques in [J-4]. See also (2.1.1).)

(1.2.1) Equivariant derived categories (characteristic 0). (See, for example, [B-L].) Let $D_{b}(E G \times \underset{G}{E})$

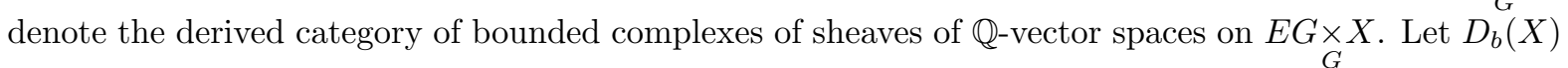
and $D_{b}(E G \times X)$ denote the corresponding derived categories on $X$ and $E G \times X$. Let $\pi: E G \times X \rightarrow$ $E G \underset{G}{\times} X$ be the quotient, and $\psi: E G \times X \rightarrow X$ the second projection. For an interval $I=[a, b]$ where $a<b$ are integers, we let $D_{I}^{G}(X)$ be the category whose objects are triples $\left(K, K_{0}, \phi\right)$ where $K \in D_{b}(E G \underset{G}{\times} X), K_{0} \in D_{b}(X)$ such that

$\mathcal{H}^{n}(K)=0$ for $n<a$ or $n>b$, and

$\phi: \pi^{*}(K) \cong \psi^{*}\left(K_{0}\right)$ is an isomorphism in $D_{b}(E G \times X)$.

A morphism between two such triples $\left(K, K_{0}, \phi\right)$ and $\left(K^{\prime}, K_{0}^{\prime}, \phi^{\prime}\right)$ is given by a pair of morphisms $K \rightarrow K^{\prime}$ in $D_{b}(E G \times X), K_{0} \rightarrow K_{0}^{\prime}$ in $D_{b}(X)$ which are compatible with the given isomorphisms $\phi$ and 
$\phi^{\prime}$. We let $D_{b}^{G}(X)$ denote the union of all the full sub-categories $D_{I}^{G}(X)$ for all intervals $I$. In case $X$ is a point, $D_{b}^{G}(X)$ is equivalent to the full sub-category of complexes $K \in D_{b}(B G)$ such that all the cohomology sheaves $\mathcal{H}^{n}(K)$ are locally constant. If moreover $G$ is connected one may in fact require the cohomology sheaves $\mathcal{H}^{n}(K)$ to be constant, because $B G$ is simply connected. In case $X$ is a point, we will denote the resulting equivariant derived category by $D_{b}^{G}(p t)$. One may define the equivariant derived category $D_{+}^{G}(X)$ similarly, by using intervals $I$ with $b=\infty$.

(1.2.2) Equivariant local systems (characteristic 0). A local system on $X$ will denote a locally constant sheaf of $\mathbb{Q}$-vector spaces. A $G$-equivariant local system on $X, \mathcal{L}$, is a locally constant sheaf of $\mathbb{Q}$-vector spaces on $E G \times X$ which belongs to the equivariant derived category $D_{b}^{G}(X)$ defined above.

The local systems on $X$ correspond to representations of the fundamental group $\pi_{1}\left(X, x_{o}\right)$ where $x_{o}$ is a fixed point of $X$. Similarly, the $G$-equivariant local systems on a $G$-variety $X$ correspond to representations of the fundamental group $\pi_{1}\left(E G \times X, x_{o}\right)$.

If $X$ is connected, the fundamental groups above are independent of the choice of the base point $x_{o}$. The only occasion where we consider non-connected varieties in the above context will be as the connected components of the orbits of a non-connected group $G$ : in this case, the connected components are all isomorphic and hence once again the fundamental group above is independent of the choice of the base point.

(1.2.3) Suppose $\pi_{1}\left(E G \underset{G}{\times} X, x_{o}\right)$ acts on the stalk $\mathcal{L}_{x_{o}}$ of a local system $\mathcal{L}$ through a finite quotient group $F$. This representation of $F$ splits into the sum of irreducible representations: therefore, in this case, $\mathcal{L}$ is semi-simple as a $G$-equivariant local system. For example, suppose $X$ is a unique orbit $G x_{o}$; then $\pi_{1}\left(E G \underset{G}{\times} X, x_{o}\right) \cong \pi_{1}\left(B G_{x_{o}}\right) \cong G_{x_{o}} / G_{x_{o}}^{0}$. Thus, every $G$-equivariant local system $\mathcal{L}$ on $X$ corresponds to a representation of the finite group $G_{x_{o}} / G_{x_{o}}^{0}$ and is therefore semi-simple.

(1.3.1) Next we will consider the corresponding situation in positive characteristics, so as to provide the correct context for formulating the main results in the paper in that setting. The main difference will be that we adopt the definition of $E G, B G$ and $E G \times \underset{G}{X}$ as simplicial schemes defined in the usual manner. (See $[\mathrm{Fr}]$ pp. 8-9.) Observe that now $B G_{0}=\operatorname{Spec}(k)$ and we will call this the base point of $B G$ : this will be denoted $*$. The above simplicial schemes will be provided with the following étale topology. Let $X_{\bullet}$ denote a simplicial scheme. Now $E t\left(X_{\bullet}\right)$ will denote the category whose objects are étale maps $u: U \rightarrow X_{n}$ for some $n$; a morphism $u: U \rightarrow X_{n}$ to $v: V \rightarrow X_{m}$ will denote a map $w: U \rightarrow V$ lying over some structure map $X_{n} \rightarrow X_{m}$. Now a sheaf $F$ on $E T\left(X_{\bullet}\right)$ is given by a collection of sheaves $\left\{F_{n} \mid n\right\}$, with $F_{n}$ a sheaf on $E t\left(X_{n}\right)$ provided with maps $\phi_{\alpha}: \alpha^{*}\left(F_{m}\right) \rightarrow F_{n}$ for any structure map $\alpha: X_{n} \rightarrow X_{m}$. (These maps are required to satisfy an obvious compatibility condition. See [Fr] p.14.) This definition applies to abelian sheaves as well as $l$-adic sheaves.

(1.3.2) Equivariant derived categories (positive characteristics) (See [J-2] section 6). Next assume $X$ • is the simplicial scheme $E G \times X$ associated to the action of $G$ on $X$. A sheaf $F$ on $E G \times X$ will be called equivariant if the above structure maps $\left\{\phi_{\alpha} \mid \alpha\right\}$ are isomorphisms. The category of equivariant sheaves is an abelian sub-category closed under extensions in the category of all sheaves on $E G \times X$; therefore one defines $D_{b}^{G}(X)$ to be the full sub-category of $D_{b}(E G \times X)$ consisting of complexes $K$ whose cohomology sheaves are all equivariant.

(1.3.3) G-equivariant local systems in positive characteristics. A $G$-equivariant local system on $X$ is a $G$-equivariant $l$-adic sheaf $F=\left\{F_{n} \mid n\right\}$ on $E G \times \underset{G}{ } X$ so that $F_{0}$ is a lisse sheaf on $X$ i.e. each term of the inverse system $F_{0}=\left\{F_{0, \nu} \mid \nu\right\}$ is locally constant. These correspond to $l$-adic representations of the étale fundamental group $\pi_{1}(E G \times X, \bar{x})$ (where $\bar{x}$ is a geometric point of $X$ ): this correspondence sends a $G$-equivariant local system to its stalk at the geometric point $\bar{x}$. To keep the notation uniform, we will identify geometric points with points: i.e. $x$ will be denote $\bar{x}$ as well.

(1.3.4) Let $\mathcal{L}$ be a $G$-equivariant $l$-adic local system on $E G \times X$ such that $\pi_{1}\left(E G \times X, x_{o}\right)$ acts on the stalk $\mathcal{L}_{x_{o}}$ through a finite quotient group $F$. Then the local system $\mathcal{L}$ corresponds to a representation of 
$F$ on the $\mathbb{Q}_{l}$-vector space associated to $\mathcal{L}_{x_{o}}$, which splits up into the sum of irreducible representations. Since the group $F$ is finite, one may show by standard arguments that each of the summands corresponds to an irreducible $l$-adic representation of $F$ and therefore to a $G$-equivariant irreducible local system on $E G \times X$. (The key observations are the following: let $V$ denote a finite dimensional $\mathbb{Q}_{l}$ vector space with a representation of $F$. Since $F$ is finite, one may find a finitely generated $\mathbb{Z}_{l}$-submodule $M$ of $V$ that is stable by $F$ and that generates $V$ as a $\mathbb{Q}_{l}$-module. Now $M$ defines an $l$-adic representation of $F$ whose inverse limit tensored with $\mathbb{Q}_{l}$ is the given vector space $V$. Recall also that the Hom between two $l$-adic local systems $L=\left\{L_{\nu} \mid \nu\right\}$ and $L^{\prime}=\left\{L_{\nu}^{\prime} \mid \nu\right\}$ is defined by $\operatorname{Hom}\left(L, L^{\prime}\right)=\lim _{\infty \leftarrow \nu} \operatorname{Hom}\left(L_{\nu}, L_{\nu}^{\prime}\right) \otimes_{\mathbb{Z}_{l}} \mathbb{Q}_{l}$ so that local systems that are torsion are identified with the local system 0.)

(1.3.4.*)It follows that, under the hypothesis that $\pi_{1}\left(\underset{G}{E} \times \underset{X}{\times} x_{o}\right)$ acts on the stalk $\mathcal{L}_{x_{o}}$ through a finite group, the $G$-equivariant local system $\mathcal{L}$ is semi-simple.

The above discussion should serve as a dictionary for translating the main results and proofs to positive characteristics.

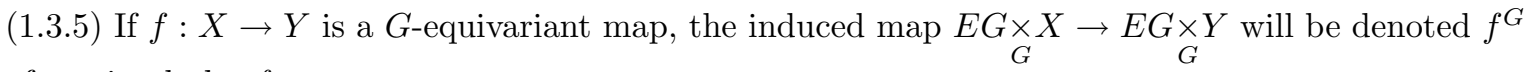
or often simply by $f$.

(1.3.6) We define equivariant hypercohomology as follows. Let $K \in D_{+}^{G}(X)$. We let $\mathbb{H}_{G}^{*}(X ; K)=$ $\mathbb{H}^{*}(E G \underset{G}{\times} X ; K)$, where $\mathbb{H}^{*}$ denotes hypercohomology.

(1.3.7) Perverse t-structures and perverse cohomology. One defines a perverse -structure on equivariant derived categories as in [B-B-D]. See [B-L] or [J-2] section 6 for details. Observe that the heart of the $t$-structure defines an abelian category and cohomology computed with values in this abelian category will be denoted $\mathcal{H}_{\text {perv }}^{n}$ : if $K \in D^{G}(X)$, then $\mathcal{H}_{\text {perv }}^{n}(K)$ is the $n$-th perverse cohomology object, which is an equivariant perverse sheaf. The cohomology truncation functor that kills the perverse cohomology in degrees above $n$ will be denoted $\tau_{\leq n}^{p e r v}$.

(1.4.1) We will presently recall the definition of equivariant intersection cohomology from [J-1] p. 242. Let $X$ be a $G$-variety of dimension $d$. Let $\phi=X_{-1} \subseteq X_{0} \subseteq X_{1} \subseteq X_{2} \subseteq \ldots \subseteq X_{d}=X$ denote a filtration by closed $G$-invariant subvarieties such that each $X_{i}$ is closed in $X$ and each $X_{i}-X_{i-1}$ is smooth, $i=0, \ldots, d$. Next one considers the complementary filtration $U_{1} \stackrel{j_{1}}{\longrightarrow} U_{2} \stackrel{j_{2}}{\longrightarrow} \ldots U_{d} \stackrel{j_{d}}{\longrightarrow} U_{d+1}=X$ where $U_{i}=X-X_{d-i}$ and $j_{i}$ denotes the inclusion. Now one applies the construction in (1.1.1) (or (1.3.1) in positive characteristics) to this filtration to obtain the following diagram:

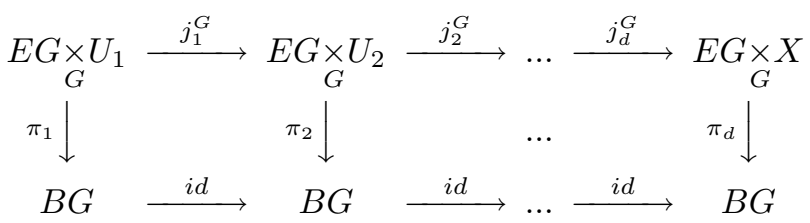

Let $\mathcal{L}$ denote a $G$-equivariant local system on $E G \times U_{1}$. We extend $\mathcal{L}$ to $E G \times X$ to obtain a complex $I C^{G}(X ; \mathcal{L})$ in $D_{b}^{G}(E G \times \underset{G}{\times})$, defined by $I C^{G}(X ; \mathcal{L})=\tau_{\leq d-1}^{G} R j_{d *}^{G} \cdots \tau_{\leq 0} R j_{0 *}(\mathcal{L})$ (see [J-1] for details). This is the equivariant intersection cohomology complex (with respect to the middle perversity) obtained from $\mathcal{L}$. In case $\mathcal{L}$ is the constant sheaf $\underline{\mathbb{Q}}\left(\underline{\mathbb{Q}}_{l}\right.$ in positive characteristics), we will denote the corresponding complex by $I C^{G}(X)$. We define

$$
I H_{G}^{*}(X ; \mathcal{L})=\mathbb{H}_{G}^{*}\left(X ; I C^{G}(X ; \mathcal{L})\right)=\mathbb{H}^{*}\left(E G \times \underset{G}{X} ; I C^{G}(X ; \mathcal{L})\right)
$$

This is a module over $H_{G}^{*}(p t)=H^{*}(B G)$. 
We will presently summarize the main properties of equivariant intersection cohomology in the following results. (See also (1.7.1) and (1.7.2).)

(1.4.4) $I C^{G}(X ; \mathcal{L})_{0}$ is the complex in $D^{G}(X)$ characterised by the following

in the situation of $(1.2 .1), \pi^{*}\left(I C^{G}(X ; \mathcal{L})\right) \simeq \psi^{*}(I C(X ; \mathcal{L}))$ and in the situation of $(1.3 .2) I C^{G}(X ; \mathcal{L})_{0} \cong$ $I C(X ; \mathcal{L})$.

Here $I C(X ; \mathcal{L})$ is the intersection cohomology complex on $X$ obtained by starting with the locally constant sheaf $\mathcal{L}$ on $U_{1}$. This is clear from the definition of the equivariant intersection cohomology complex.

(1.4.5) Theorem. (Localization theorem in the equivariant derived category for torus actions). Let $T$ denote a torus acting on a variety $X$ and let $K \in D_{b}^{T}(X)$. Let $T_{0}$ denote a sub-torus of $T$ and let $\mathfrak{p}$ be the kernel of the restriction map $H^{*}(B T) \rightarrow H^{*}\left(B T_{0}\right)$; this is a prime ideal of $H^{*}(B T)$. Let $i: X^{T_{0}} \rightarrow X$ denote the inclusion of the fixed point subscheme of $T_{0}$. Then one obtains the following isomorphism after localization at the ideal $\mathfrak{p}$ :

$$
\mathbb{H}_{T}^{*}(X ; K)_{\mathfrak{p}} \cong \mathbb{H}_{T}^{*}\left(X^{T_{0}} ; R i^{T !} K\right)_{\mathfrak{p}}
$$

Proof. This is a generalization of the localization theorem in [J-1] (17) and [Bryl], but the proof is essentially the same. We consider the localization sequence:

$$
\cdots \rightarrow \mathbb{H}_{T}^{n}\left(X^{T_{0}} ; R i^{T !} K\right) \rightarrow \mathbb{H}_{T}^{n}(X ; K) \rightarrow \mathbb{H}_{T}^{n}\left(X-X^{T_{0}} ; K\right) \rightarrow \cdots
$$

Since localization at the prime $\mathfrak{p}$ is exact, it suffices to show that the last term is trivial after localization at $\mathfrak{p}$. Now observe that $\mathbb{H}_{T}^{*}\left(X-X^{T_{0}} ; K\right)$ is a module over $H_{T}^{*}\left(X-X^{T_{0}}\right)$. Therefore it suffices to show that the latter is trivial after localization at $\mathfrak{p}$. This follows for example from [Br-2] Appendix, Proposition 5.

(1.5) Let $T$ denote a maximal torus in $G$, let $N_{G}(T)\left(C_{G}(T)\right)$ denote its normalizer (centralizer, respectively) in $G$ and let $W=N_{G}(T) / C_{G}(T)^{0}$. Then $W$ is a finite group, since $N_{G}(T) / C_{G}(T)$ and $C_{G}(T) / C_{G}(T)^{0}$ are finite groups. We will call $W$ the Weyl group of $(G, T)$.

(1.5.1) Proposition. Let $X$ be a $G$-variety and let $\mathcal{L}$ be a $G$-equivariant local system on an open $G$-stable smooth subvariety of $X$.

(i) Then $N_{G}(T)$ acts on $I H_{T}^{*}(X ; \mathcal{L})$, the action of $C_{G}(T)^{0}$ is trivial, and one has the following isomorphisms:

$$
I H_{G}^{*}(X ; \mathcal{L}) \cong I H_{N_{G}(T)}^{*}(X ; \mathcal{L}) \cong I H_{T}^{*}(X ; \mathcal{L})^{W}
$$

as modules over $H^{*}(B G) \cong H^{*}(B T)^{W}$.

(ii) If moreover $i_{Y}: Y \rightarrow X$ is the inclusion of a $G$-stable closed subvariety, one has the isomorphisms

$$
\mathbb{H}_{G}^{*}\left(Y ; R i_{Y}^{!} I C^{G}(X ; \mathcal{L})\right) \cong \mathbb{H}_{N_{G}(T)}^{*}\left(Y ; R i_{Y}^{!} I C^{N_{G}(T)}(X ; \mathcal{L})\right) \cong \mathbb{H}_{T}^{*}\left(Y ; R i_{Y}^{!} I C^{T}(X ; \mathcal{L})\right)^{W}
$$

Proof. The map

$$
\left.\pi: E G \times \underset{G}{\times} \underset{N_{G}(T)}{\times} X\right) \rightarrow \underset{G}{E} X
$$

is a fibration with fiber $G / N_{G}(T)$. Therefore, $\pi^{*} I C^{G}(X ; \mathcal{L})$ is isomorphic to $I C^{G}\left(G \underset{N_{G}(T)}{\times} X ; \mathcal{L}\right)$ which may be identified with $I C^{N_{G}(T)}(X ; \mathcal{L})$, see [J-2] or [J-4] $(4.4)$. Moreover, we claim that the fibers of $\pi$ are acyclic; then the first isomorphism in (i) and the first isomorphism in (ii) will follow from the Leray spectral sequence and the projection formula.

For the claim, we first reduce to the case where $G$ is connected, as follows. The connected component $G^{0}$ contains $T$. We check that the map from $G^{0} / N_{G^{0}}(T)$ to $G / N_{G}(T)$ is an isomorphism. Since this map factors as $G^{0} / N_{G^{0}}(T) \rightarrow G / N_{G^{0}}(T) \rightarrow G / N_{G}(T)$, it is clearly étale. It is also obviously injective. 
We show the surjectivity as follows. Let $g$ in $G$, then $g T g^{-1}$ is a maximal torus of $G^{0}$. Thus, there exists $h$ in $G^{0}$ such that $g T g^{-1}=h T h^{-1}$. So $h^{-1} g$ is in $N_{G}(T)$. This shows that $G=G^{0} N_{G}(T)$ and therefore our map is surjective, i.e. it is an étale bijective map and hence an isomorphism of varieties.

Now we reduce to the case where $G$ is (connected and) reductive. Let $R_{u}(G)$ be the unipotent radical of $G$, with quotient $\bar{G}=G / R_{u}(G)$, a connected reductive group. We identify $T$ with its image in $\bar{G}$. Since the composition $G \rightarrow \bar{G} \rightarrow \bar{G} / N_{\bar{G}}(T)$ is surjective and clearly factors through $G \rightarrow G / N_{G}(T)$, it follows that the induced map $G / N_{G}(T) \rightarrow \bar{G} / N_{\bar{G}}(T)$ is also surjective. Clearly it is smooth with fibers $R_{u}(G) / N_{R_{u}(G)}(T)$. As a homogeneous space under a unipotent group, the latter is isomorphic to an affine space and hence is acyclic.

Now we can assume that $G$ is connected and reductive, or even semi-simple because $N_{G}(T)$ contains the center of $G$. Let $B$ be a Borel subgroup of $G$ containing $T$; then the map from $G / T$ to $G / B$ has acyclic fibers and thus $H^{*}(G / T)$ is isomorphic to $H^{*}(G / B)$. The latter is the regular representation of $W=N_{G}(T) / T$, and moreover $\left.H^{*}\left(G / N_{G}(T)\right)\right)=H^{*}(G / T)^{W}$ which proves our claim.

To prove the second isomorphism in (i), note that $I H_{N_{G}(T)}^{*}(X ; \mathcal{L})$ is isomorphic to $I H_{C_{G}(T)^{0}}^{*}(X ; \mathcal{L})^{W}$. (This follows from Theorem 1.) Moreover, the quotient $C_{G}(T)^{0} / T$ is unipotent. Thus, $I H_{C_{G}(T)^{0}}^{*}(X ; \mathcal{L})$ is isomorphic to $I H_{T}^{*}(X ; \mathcal{L})$. Now one may invoke Theorem 1 again to complete the proof. (Observe that $I C^{T}(X ; \mathcal{L})$ is the restriction of $I C^{N_{G}(T)}(X ; \mathcal{L})$ and similarly $R i_{Y}^{!} I C^{T}(X ; \mathcal{L})$ is the restriction of $R i_{Y}^{\prime} I C^{N_{G}(T)}(X ; \mathcal{L})$. Therefore the hypotheses of Theorem 1 are satisfied.)

(1.5.2) Theorem. (Degeneration of the spectral sequence in equivariant intersection cohomology). Let $X$ be a projective $G$-variety, where $G$ is connected. Let $\mathcal{L}$ denote a $G$-equivariant local system on an open dense $G$-stable smooth subvariety of $X$ such that $\mathcal{L}$ is semi-simple as a local system. Let $I C^{G}(X ; \mathcal{L})$ denote the corresponding equivariant intersection cohomology complex. Then the spectral sequence:

$$
E_{2}^{s, t}=H^{s}\left(B G ; R^{t} \pi_{*}\left(I C^{G}(X ; \mathcal{L})\right)\right) \Rightarrow I H_{G}^{s+t}(X ; \mathcal{L})
$$

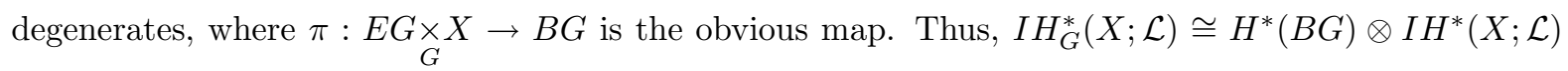
as $H^{*}(B G)$-modules.

Proof is essentially the same as in [J-1] Proposition (13) where only the case $G$ is a one dimensional torus is considered. That $G$ be connected is necessary to ensure that all local systems on $B G$ are in fact constant. Let $U$ denote an open smooth $G$-stable subvariety of $X$ on which $\mathcal{L}$ is a local system. Since $X$ is equidimensional, $U$ is the disjoint union of its connected components $U_{i}$ all of which are of the same dimension. Since $G$ is connected the $U_{i}$ are stable under the group action. Let $\mathcal{L}_{i}$ denote the $G$-equivariant local system on $U$ defined by $\mathcal{L}_{i_{\mid U_{j}}}=\mathcal{L}_{\mid U_{i}}$ if $j=i$, and $\mathcal{L}_{i_{\mid U_{j}}}=0$ otherwise. Then one may see that $I C^{G}(X ; \mathcal{L})=\oplus_{i} I C^{G}\left(X ; \mathcal{L}_{i}\right)$. Clearly each $\mathcal{L}_{i}$ is a semi-simple local system; therefore each $I C\left(X ; \mathcal{L}_{i}\right)$ and hence $I C(X ; \mathcal{L})$ is a pure perverse sheaf. Therefore, the Hard Lefschetz theorem holds for $I H^{*}(X ; \mathcal{L})$ and the same proof as in $[\mathrm{J}-1]$ Proposition (13) applies.

(1.5.3) Theorem. Let $X$ denote a projective variety provided with the action of a torus $T$ and let $\mathcal{L}$ denote a $T$-equivariant local system on an open smooth $T$-stable subvariety of $X$. Assume that $\mathcal{L}$ is semi-simple as a local system. Let $i: X^{T} \rightarrow X$ denote the inclusion of the fixed point subscheme. Now one obtains the isomorphisms after inverting all non-zero elements of $H^{*}(B T)$ (i.e. on localization at the prime ideal (0)):

$$
I H_{T}^{*}(X ; \mathcal{L})_{(0)} \cong H^{*}(B T)_{(0)} \otimes I H^{*}(X ; \mathcal{L}) \cong H^{*}(B T)_{(0)} \otimes \mathbb{H}^{*}\left(X^{T} ; R i^{\prime} I C(X ; \mathcal{L})\right) .
$$

In particular, if $I H^{n}(X ; \mathcal{L})=0$ for all odd $n$ and $x$ is an isolated fixed point of $T$, then $I H_{x}^{n}(X ; \mathcal{L})=0$ for all odd $n$.

Proof. The first isomorphism follows from (1.5.2) by localizing at (0). By the localization theorem in (1.4.5), one has the isomorphism:

$$
I H_{T}^{*}(X ; \mathcal{L})_{(0)} \cong \mathbb{H}_{T}^{*}\left(X^{T} ; R i^{!} I C^{T}(X ; \mathcal{L})\right)_{(0)} .
$$


Now $\mathbb{H}_{T}^{*}\left(X^{T} ; R i^{!} I C^{T}(X ; \mathcal{L})\right)_{(0)} \cong H^{*}(B T)_{(0)} \otimes \mathbb{H}^{*}\left(X^{T}, R i^{!} I C^{T}(X ; \mathcal{L})\right)_{(0)}$.

Next let $i_{x}: x \rightarrow X^{T}$ be the inclusion of an isolated fixed point of $T$. Then $R i^{!} I C^{T}(X ; \mathcal{L})$ breaks up into the sum of two complexes one of which is

$$
R i_{x}^{!} I C^{T}(X ; \mathcal{L}) \cong D i_{x}^{*} D\left(I C^{T}(X ; \mathcal{L})\right) \cong\left(i_{x}^{*} I C^{T}\left(X ; \mathcal{L}^{\vee}\right)\right)^{\vee}[-2 d]
$$

where $d$ is the dimension of $X$. This proves the last assertion of the theorem.

We will now recall a few results from equivariant derived categories as in [J-2].

Let $H$ denote a closed subgroup of $G$ and let $X$ be a $H$-variety. Then we have a closed immersion

$$
j_{X}: \underset{H}{E \underset{H}{X} X} \underset{G}{\operatorname{Ex}} \underset{H}{\times}(G \times \underset{H}{\times})
$$

induced by the map $X \rightarrow \underset{H}{G \times X}$ sending $x$ to the class of $(e, x)$. Let $K \in D_{b}^{G}(G \underset{H}{\times} X)$. Then $j_{X}^{*}(K)$ is a

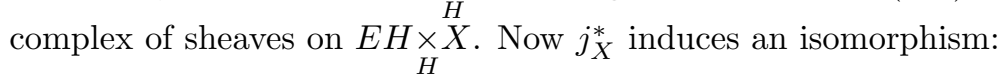

$$
\mathbb{H}_{H}^{*}\left(X ; j_{X}^{*}(K)\right) \stackrel{\cong}{\longrightarrow} \mathbb{H}_{G}^{*}(G \times \underset{H}{\times} ; K)
$$

See $[\mathrm{J}-2]$ section 6 for a proof.

(1.6.2) Assume in addition to the hypothesis in (1.6.1) that $X$ is a point $x$. In this case the cohomology sheaves of $K$ are equivariant on the $G$-orbit $\underset{H}{G \times x} \cong G / H$ and hence locally constant. Therefore, we may identify $j_{x}^{*} K$ with $R j_{x}^{!} K\left[2 d_{x}\right]$ where $d_{x}$ is the dimension of the $G$-orbit $\underset{H}{G \times x}$. Now (1.6.1) provides the isomorphism:

$$
\mathbb{H}^{*+2 d_{x}}\left(B H ; R j_{x}^{!} K\right) \cong \mathbb{H}_{H}^{*+2 d_{x}}\left(x ; R j_{x}^{!} K\right) \stackrel{\cong}{\longrightarrow} \mathbb{H}_{G}^{*}(G / H ; K) .
$$

(1.7.0) Reduction to the constant local system by normalization.

In the rest of this section we discuss a technique whereby we are able to reduce consideration of arbitrary local systems to a constant local system; this simplifies much of the discussion in Section 3.

Let $G$ be connected and let $\pi: \tilde{X} \rightarrow X$ be a $G$-equivariant finite map between $G$-varieties. There will be two main examples of this situation we consider:

(i) $\pi$ is the normalization of $X$ and

(ii) $G$ has a dense orbit $G x$ on $X$ (so that $X$ is irreducible) and $\tilde{X}$ is the normalization of $X$ in the function field $k\left(G / G_{x}^{0}\right)$.

(1.7.1) Proposition. Assume the situation of (1.7.0). Then the following hold:

(a) If $G$ has finitely many orbits on $X$, the same holds for the $G$-action on $\tilde{X}$.

(b) If $\mathcal{S}$ is any affine locally closed subvariety in $X$, its inverse image $\tilde{\mathcal{S}}$ in $\tilde{X}$ is affine and locally closed.

(c) Let $\mathcal{S}$ be as in (b). If, moreover, $\mathcal{S}$ is invariant under the action of a subtorus $T$ of $G$, so is $\tilde{\mathcal{S}}$. If $x$ is an attractive fixed point for the $T$-action on $\mathcal{S}$, and $\tilde{x}$ is a lift of $x$ to $\tilde{X}$, then $\tilde{x}$ is an attractive fixed point on the connected component of $\tilde{\mathcal{S}}$ containing $\tilde{x}$.

(d) Assume the situation as in (a). If, moreover, every $G$-orbit in $X$ admits an attractive slice, then the same holds for $\tilde{X}$.

Proof. The first assertion is clear since the map $\pi$ is finite. (In fact the inverse image of a $G$-orbit in $X$ is the disjoint union of finitely many $G$-orbits in $\tilde{X}$.) Now we consider the second assertion. Let 
$\pi_{\mathcal{S}}: \tilde{\mathcal{S}} \rightarrow \mathcal{S}$ denote the restriction of the map $\pi$. Then the morphism $\pi_{\mathcal{S}}$ is finite, and hence affine. Since $\mathcal{S}$ is affine, it follows that $\tilde{\mathcal{S}}$ is affine as well. That $\tilde{\mathcal{S}}$ is locally closed is clear.

Now we consider the third assertion. Clearly each connected component of $\tilde{\mathcal{S}}$ is stable under the action of $T$. Let $\lambda$ denote a one-parameter subgroup chosen as in (0.2). Then the action of $\lambda$ on the ring of regular functions $k[\mathcal{S}$ ] defines a non-negative grading such that the degree 0 component is one dimensional. Since $k[\tilde{\mathcal{S}}]$ is integral over $k[\mathcal{S}]$, it has a non-negative grading and the degree 0 component is finite dimensional. This proves (c). We let $\tilde{S}_{\tilde{x}}$ denote the connected component of $\tilde{\mathcal{S}}$ containing $\tilde{x}$. Observe that the map $G \times \tilde{\mathcal{S}}_{\tilde{x}} \rightarrow \tilde{X}$ factors through $G \times \tilde{\mathcal{S}}_{\tilde{x}} \rightarrow G \times \pi^{-1}(\mathcal{S})$, an open immersion, followed by the map $G \times \pi^{-1}(\mathcal{S}) \rightarrow \tilde{X}$. The last map is obtained by base change from the smooth map $G \times \mathcal{S} \rightarrow X$ and therefore is smooth. This proves the last assertion.

(1.7.2) Proposition (i) Let $\pi: \tilde{X} \rightarrow X$ denote the normalization of a $G$-variety. Let $\mathcal{L}$ denote a $G$-equivariant local system on an open $G$-stable smooth sub-variety of $X$ and let $\tilde{\mathcal{L}}=\pi^{*}(\mathcal{L})$. Then $R \pi_{*} I C^{G}(\tilde{X} ; \tilde{\mathcal{L}})=I C^{G}(X ; \mathcal{L})$ and therefore

$$
I H_{G, Y}^{*}(X ; \mathcal{L}) \cong I H_{G, \tilde{Y}}^{*}(\tilde{X} ; \tilde{\mathcal{L}})
$$

for any $G$-stable closed sub-variety $Y$ of $X$ and $\tilde{Y}=\pi^{-1}(Y)$.

(ii) Assume that $X$ contains a dense orbit $G x$ and let $\pi: \tilde{X} \rightarrow X$ denote the normalization of $X$ in the function field of $k\left(G / G_{x}^{0}\right)$. Then

$$
\pi_{*}\left(I C^{G}(\tilde{X})\right)=\underset{\chi}{\oplus} \operatorname{dim}(\chi) I C^{G}\left(X ; \mathcal{L}_{\chi}\right) \text { and } I H_{G, \tilde{Y}}^{*}(\tilde{X}) \cong \underset{\chi}{\oplus} \operatorname{dim}(\chi) I H_{G, Y}^{*}\left(X ; \mathcal{L}_{\chi}\right)
$$

Here $\mathcal{L}_{\chi}$ is the irreducible local system on $G x$ corresponding to the irreducible character $\chi$ of $G_{x} / G_{x}^{0}$ and the sum varies over all such characters. Moreover, this decomposition of the local intersection cohomology is natural with respect to locally closed $G$-stable subvarieties $Y$ of $X$ and $\tilde{Y}=\pi^{-1}(Y)$.

(iii) In the situation of (i) or (ii), let $\mathcal{S}$ be an attractive slice in $X$ at the fixed point $x$ under a subtorus $T$. Let $\pi^{-1}(x)=\left\{\tilde{x}_{1}, \ldots, \tilde{x}_{n}\right\}$ and let $\tilde{\mathcal{S}}=\sqcup_{i} \tilde{\mathcal{S}}_{\tilde{x}_{i}}$ with $\tilde{\mathcal{S}}_{\tilde{x}_{i}}$ denoting the connected component of $\tilde{\mathcal{S}}$ containing $\tilde{x}_{i}$. Let $i_{x}: E T \underset{T}{\times x} \rightarrow \underset{T}{\times} \underset{T}{\mathcal{S}}, i_{\tilde{x}_{i}}: E T \underset{T}{\operatorname{x}} \tilde{x}_{i} \rightarrow E T \underset{T}{\times \tilde{\mathcal{S}}_{\tilde{x}_{i}}}$ denote the inclusion maps. Then

$$
i_{x}^{*} I C^{T}(\mathcal{S} ; \mathcal{L}) \cong \underset{i}{\oplus i} i_{\tilde{x}_{i}}^{*} I C^{T}\left(\tilde{\mathcal{S}}_{\tilde{x}_{i}} ; \tilde{\mathcal{L}}\right)
$$

in the situation of (i), whereas

$$
\underset{\chi}{\oplus} \operatorname{dim}(\chi) i_{x}^{*} I C^{T}\left(\mathcal{S} ; \mathcal{L}_{\chi}\right) \simeq \underset{i}{\oplus} i_{\tilde{x}_{i}}^{*} I C^{T}\left(\tilde{\mathcal{S}}_{\tilde{x}_{i}}\right)
$$

in the situation of (ii).

Proof (i) Observe that the map $\pi$ is birational. It follows readily that $R \pi_{*} I C^{G}(\tilde{X} ; \tilde{\mathcal{L}})=I C^{G}(X: \mathcal{L})$. By taking $G$-equivariant hypercohomology on $X$ (with supports in $Y$ ), we therefore obtain the isomorphism:

$$
I H_{G, Y}^{*}(X ; \mathcal{L}) \cong I H_{G, \tilde{Y}}^{*}(\tilde{X} ; \tilde{\mathcal{L}})
$$

This proves (i). Next we consider (ii). Let $\pi_{0}: \tilde{U}=\pi^{-1}(G x) \rightarrow G x$ denote the map induced by

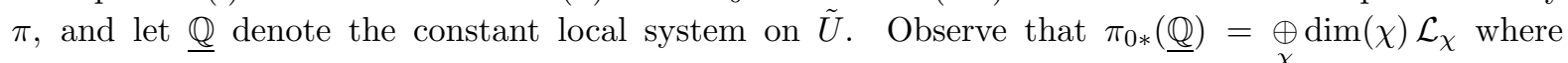
$\mathcal{L}_{\chi}$ is the irreducible local system on $G x$ corresponding to the character $\chi$ of $G_{x} / G_{x}^{0}$. Therefore $R \pi_{*} I C^{G}(\tilde{X})=\oplus_{\chi} \operatorname{dim}(\chi) I C^{G}\left(X ; \mathcal{L}_{\chi}\right)$. The remaining assertions in (ii) are clear by taking $G$-equivariant hypercohomology on $X$.

Now we consider (iii) in the situation of (ii). Consider the cartesian square

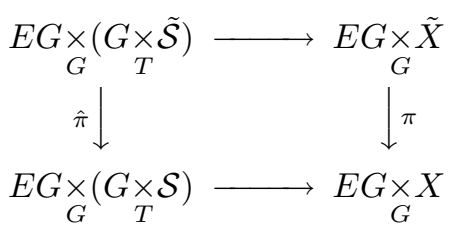


where the map $\hat{\pi}$ is induced by $\pi$. Therefore, by proper base change, one obtains that

$$
\left.\hat{\pi}_{*}\left(I C^{G}(G \times \underset{T}{\times \mathcal{S}})\right) \cong \underset{\chi}{\oplus} \operatorname{dim}(\chi) I C^{G}\left(G \times \underset{T}{*} ; \mathcal{L}_{\chi}\right)\right)
$$

(Here we have used $\mathcal{L}_{\chi}$ to also denote the pull-back of the local system $\mathcal{L}_{\chi}$ to $G \times \mathcal{T}$ ).) Under the equivalence of derived categories $D^{G}(\underset{T}{\underset{\mathcal{S}}{*}}) \simeq D^{T}(\tilde{\mathcal{S}})$ and $D^{G}(G \times \mathcal{S}) \simeq D^{T}(\mathcal{S})$, the isomorphism in (1.7.2.1) corresponds to

$$
\tilde{\pi}_{*}\left(I C^{T}(\tilde{\mathcal{S}})\right) \cong \underset{\chi}{\oplus} \operatorname{dim}(\chi) I C^{T}\left(\mathcal{S} ; \mathcal{L}_{\chi}\right)
$$

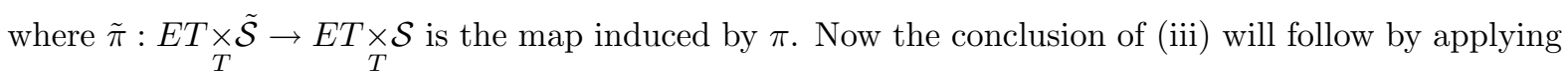
base change to the cartesian square:

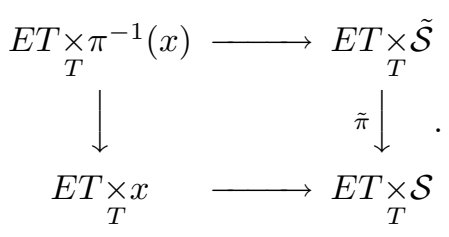

The argument in the situation of (i) is similar. (In the place of $(1.7 .2 .1)((1.7 .2 .2))$ we obtain $\hat{\pi}_{*}\left(I C^{G}(G \times \tilde{\mathcal{S}} ; \tilde{\mathcal{L}})\right) \cong$ $I C^{G}(\underset{T}{\operatorname{So}} ; \mathcal{L})\left(\tilde{\pi}_{*}\left(I C^{T}(\tilde{\mathcal{S}} ; \tilde{\mathcal{L}})\right) \cong I C^{T}(\mathcal{S} ; \mathcal{L})\right.$, respectively $)$.

We will consider only the case of characteristic zero explicitly throughout the rest of the paper.

\section{Proof of Theorem 1}

We will prove the first three statements of Theorem 1 first. Let $K \in D_{b}^{G}(X)$ and let $\pi: E G \times \underset{G}{E} X \rightarrow B \bar{G}$ denote the obvious map. We will presently show that $R^{n} \pi_{*}(K)$ is a locally constant sheaf on $B \bar{G}$ for

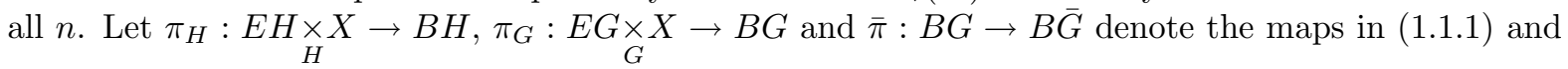
(1.1.2). Observe that $\pi$ factors as $\bar{\pi} \circ \pi_{G}$. By replacing $K$ by $R \pi_{G *} K$ we may assume $X$ is a point i.e. $E H \underset{H}{\times} X=B H, \underset{G}{E} \underset{G}{\times} X=B G$ and $\pi=\bar{\pi}$. Now let $\gamma: I=[0,1] \rightarrow B \bar{G}$ denote a path; let $F_{I}, F_{0}$ and $F_{1}$ denote the pull backs under $\pi$ over $I, 0$ and 1 respectively. Now one obtains spectral sequences:

$$
\begin{aligned}
& E_{2}^{s, t}(I)=H^{s}\left(F_{I}, \mathcal{H}^{t}(K)_{\mid F_{I}}\right) \Rightarrow \mathbb{H}^{s+t}\left(F_{I}, K_{\mid F_{I}}\right), \\
& E_{2}^{s, t}(0)=H^{s}\left(F_{0}, \mathcal{H}^{t}(K)_{\mid F_{0}}\right) \Rightarrow \mathbb{H}^{s+t}\left(F_{0}, K_{\mid F_{0}}\right), \\
& E_{2}^{s, t}(1)=H^{s}\left(F_{1}, \mathcal{H}^{t}(K)_{\mid F_{1}}\right) \Rightarrow \mathbb{H}^{s+t}\left(F_{1}, K_{\mid F_{1}}\right) .
\end{aligned}
$$

Clearly the inclusions of $F_{0}$ and $F_{1}$ in $F_{I}$ induce maps of these spectral sequences. Since the cohomology sheaves of $K$ are locally constant, (by choosing the path to lie in a small enough open set in $B \bar{G}$ ) and the fibration $\bar{\pi}$ is locally trivial, it follows that one obtains an isomorphism at the $E_{2}$ terms and thereby an isomorphism of the abutments. Thus, each $R^{n} \pi_{*}(K)$ is locally constant.

Now it suffices to obtain the identification of the stalks of the above sheaf at the base point $p$ in $B \bar{G}$. We go back to the general case where $X$ is no longer a point.

Since $R \pi_{*} K=R \bar{\pi}_{*} R \pi_{G *}(K)$, we have

$$
R^{n} \pi_{*}(K)_{p} \cong R^{n} \bar{\pi}_{*}\left(R \pi_{G *} K\right)_{p} \cong \mathbb{H}^{n}\left(B H ; R \pi_{G *} K_{\mid B H}\right) \cong \mathbb{H}^{n}\left(B H ; R \pi_{H *}\left(K_{\mid E H \underset{H}{\times} X}\right)\right) \cong \mathbb{H}_{H}^{n}\left(X ; K_{\mid E H \times} \times \underset{H}{ }\right) .
$$


The second isomorphism follows from the observation that the map $\bar{\pi}$ is a locally trivial fibration with fibers $B H$. The third isomorphism is clear by observing that that both $\pi_{G}$ and $\pi_{H}$ are locally trivial fibrations with fiber $X$. The equivalence of categories between locally constant sheaves on $B \bar{G}$ and representations of $\pi_{1}(B \bar{G})=\bar{G}$, now provides an action of $\bar{G}$ on the stalks of $R^{n} \pi_{*}(K)$ that is natural in $K$. Finally the Leray spectral sequence for the map $\pi$ provides the spectral sequence:

$$
E_{2}^{s, t}=H^{s}\left(B \bar{G} ; R^{t} \pi_{*}(K)\right) \Rightarrow \mathbb{H}_{G}^{s+t}(X ; K) .
$$

This spectral sequence degenerates since $\bar{G}$ is a finite group and provides the isomorphism $\mathbb{H}_{G}^{*}(X ; K)=$ $\left(\mathbb{H}_{H}^{*}(X ; K)\right)^{\bar{G}}$.

The last statement in (iii) may be obtained as follows. Since $F$ acts trivially on $X$, we obtain $E G \times X \cong B F \times\left(E G^{0} \times X\right)$ and therefore an induced map $\tilde{\pi}: E G \times X \rightarrow E G^{0} \times X$. Now $G^{0}$

$$
H_{G}^{*}(X ; K) \cong \mathbb{H}^{*}\left(B F ; R \pi_{*}(K)\right) \cong \mathbb{H}_{G^{0}}^{*}\left(X ; R \tilde{\pi}_{*}(K)\right)
$$

Observe that $\mathbb{H}^{*}\left(B F ; R \pi_{*}(K)\right) \cong H_{G^{0}}^{*}(X ; K)^{F}$ while $\mathbb{H}_{G^{0}}^{*}\left(X ; R \tilde{\pi}_{*}(K)\right) \cong H_{G^{0}}^{*}\left(X ; K^{F}\right)$. This completes the proof of (iii) in Theorem 1.

(2.1.0) Observe from the above proof that each $R^{n} \bar{\pi}_{*}(K)$ is a local system on $B \bar{G}$.

(2.1.1) It will become necessary to develop the techniques in (2.1.1) through (2.2.5) in order to establish the last statement in Theorem 1. Establishing the corresponding results in positive characteristics involves defining the derived functor $R \bar{\pi}_{*}$ so that its stalks may be identified with the equivariant hypercohomology with respect to the group $H$. Therefore we leave this to the paper [J-4] by the second author.

(2.1.2) Recall that the map $\bar{\pi}: B G \rightarrow B \bar{G}$ is a fibration with fiber $B H$. If $\mathbb{Q}$ is the constant sheaf on $B G$, then $R \bar{\pi}_{*}(\underline{\mathbb{Q}})$ is a complex of sheaves on $B \bar{G}$. The multiplication $\underline{\mathbb{Q}} \otimes \mathbb{Q} \longrightarrow \underline{\mathbb{Q}}$ induces an associative pairing: $R \bar{\pi}_{*}(\underline{Q}) \otimes R \bar{\pi}_{*}(\underline{\mathbb{Q}}) \rightarrow R \bar{\pi}_{*}(\underline{\mathbb{Q}})$. This shows $R \bar{\pi}_{*}(\underline{\mathbb{Q}})$ is a sheaf of differential graded algebras on $B \bar{G}$.

(2.1.3) Let $\operatorname{BiMod}\left(B \bar{G} ; R \bar{\pi}_{*}(\mathbb{Q})\right)$ denote the category of sheaves of bi-modules over the sheaf of differential graded algebras $R \bar{\pi}_{*}(\mathbb{Q})$ on $B \bar{G}$. An object in this category is a complex of sheaves $M$ on $B \bar{G}$ provided with (coherently) associative pairings : $R \bar{\pi}_{*}(\underline{\mathbb{Q}}) \otimes M \rightarrow M$ and $M \otimes R \bar{\pi}_{*}(\underline{\mathbb{Q}}) \rightarrow M$ that make the obvious diagrams commute. (See $[\mathrm{K}-\mathrm{M}]$ for details on differential graded algebras and modules over them.)

(2.1.4) Given $K \in D_{b}^{G}(B G)$, the associative pairings $\underline{\mathbb{Q}} \otimes K \rightarrow K$ and $K \otimes \underline{\mathbb{Q}} \rightarrow K$ show that $R \bar{\pi}_{*}(K)$ belongs to $\operatorname{BiMod}\left(B \bar{G} ; R \bar{\pi}_{*}(\underline{\mathbb{Q}})\right)$.

(2.1.5) Let $\Delta[1] \otimes \mathbb{Q}$ denote the normalization of the simplicial abelian group given by $n \rightarrow \Delta[1]_{n} \otimes \mathbb{Q}$. We will define two maps $f, g: K \rightarrow K^{\prime}$ in $\operatorname{BiMod}\left(B \bar{G} ; R \bar{\pi}_{*}(\underline{\mathbb{Q}})\right)$ to be homotopic if there exists a map $H: K \otimes \Delta[1] \otimes \underline{\mathbb{Q}} \rightarrow K^{\prime}$ so that $f=H \circ d_{0}$ and $g=H \circ d_{1}$, with $d_{i}: K \cong K \otimes \Delta[0] \otimes \underline{\mathbb{Q}} \rightarrow K \otimes \Delta[1] \otimes \underline{\mathbb{Q}}$, $i=0,1$, being the obvious map. The associated homotopy category is denoted $\operatorname{HBi} \bar{M} \operatorname{od}\left(B \bar{G} ; R \bar{\pi}_{*}(\mathbb{Q})\right)$. The corresponding derived category is obtained from $\operatorname{HBiMod}\left(B \bar{G} ; R \bar{\pi}_{*}(\underline{\mathbb{Q}})\right)$ by inverting maps that induce isomorphisms on cohomology. Its full sub-category consisting of complexes that are bounded below (and whose cohomology sheaves are $\bar{G}$-equivariant) will be denoted $D_{+}\left(\operatorname{BiMod}\left(B \bar{G} ; R \bar{\pi}_{*}(\underline{\mathbb{Q}})\right)\right)$ $\left(D_{+}^{\bar{G}}\left(B i-\operatorname{Mod}\left(B \bar{G} ; R \bar{\pi}_{*}(\underline{\mathbb{Q}})\right)\right)\right.$, respectively $)$.

Next let $M, N$ in $D_{+}^{\bar{G}}\left(\operatorname{BiMod}\left(B \bar{G} ; \quad R \bar{\pi}_{*}(\underline{\mathbb{Q}})\right)\right)$. Then there exists a spectral sequence:

$$
E_{2}^{s, t}=\mathcal{E} x t_{\mathcal{H}^{*}\left(R \bar{\pi}_{*}(\underline{\mathbb{Q}})\right)}^{s, t}\left(\mathcal{H}^{*}(M), \mathcal{H}^{*}(N)\right) \Rightarrow \mathcal{H}^{s+t}\left(\mathcal{R} \mathcal{H}^{o m} m_{R \bar{\pi}_{*}(\underline{\mathbb{Q}})}(M, N)\right.
$$

If moreover at each point $\bar{x}$ of $B \bar{G}, \mathcal{H}^{*}(M)_{\bar{x}}$ is a free module over $\mathcal{H}^{*}\left(R \bar{\pi}_{*}(\underline{\mathbb{Q}})\right)_{\bar{x}} \cong H^{*}(B H ; \mathbb{Q})$, then the above spectral sequence degenerates and $E_{2}^{s, t}=0$ for all $s>0$. The spectral sequence is established in [K-M] Theorem (7.3), Part V. 
Next we define functors

$$
L \bar{\pi}^{*}: D_{+}^{\bar{G}}\left(\operatorname{BiMod}\left(B \bar{G} ; R \bar{\pi}_{*}(\underline{\mathbb{Q}})\right)\right) \rightarrow D_{+}^{G}(B G)
$$

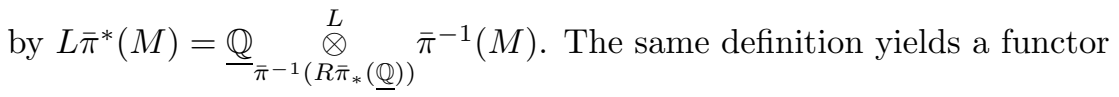

$$
L \bar{\pi}^{*}: D_{b}\left(\operatorname{BiMod}\left(B \bar{G} ; R \bar{\pi}_{*}(\underline{\mathbb{Q}})\right)\right) \rightarrow D_{+}(B G) .
$$

(See $[\mathrm{K}-\mathrm{M}]$ part III for a definition of the above derived functors in a somewhat more general setting.)

(2.2.3) Proposition. Assume in addition to the above hypotheses that the group $H$ is connected.

(i) If $K \in D_{b}^{G}(B G)$, there exists a map $L \bar{\pi}^{*} R\left(\bar{\pi}_{*} K\right) \rightarrow K$ that is a quasi-isomorphism and is natural in $K$.

(ii) The functor $R \bar{\pi}_{*}: D_{b}^{G}(B G) \rightarrow D_{+}^{\bar{G}}\left(\operatorname{BiMod}\left(B \bar{G} ; R \bar{\pi}_{*}(\underline{\mathbb{Q}})\right)\right)$ is fully faithful.

(iii) If $K, K^{\prime} \in D_{b}^{G}(B G)$, the map in (i) induces a quasi-isomorphism:

$$
R \bar{\pi}_{*}\left(\mathcal{R} \mathcal{H o m}\left(K, K^{\prime}\right)\right) \rightarrow \mathcal{R} \mathcal{H o m} \bar{\pi}_{*}(\underline{\mathbb{Q}})\left(R \bar{\pi}_{*}(K), R \bar{\pi}_{*}\left(K^{\prime}\right)\right) .
$$

(The two $\mathcal{R}$ Hom denote derived functors of the appropriate internal Homs.)

Proof. Observe that $L \bar{\pi}^{*}$ is the left adjoint to the functor $R \bar{\pi}_{*}$. Therefore the naturality of the map in (i) is clear. To show it is a quasi-isomorphism one proceeds as follows. From the proofs of (i) through (iii) of Theorem 1 above, it follows that each $R^{n} \bar{\pi}_{*}(K)$ is a local system on $B \bar{G}$. Let $\bar{x}$ denote a point of $B \bar{G}$, let $i_{\bar{x}}: \bar{x} \rightarrow B \bar{G}$ and let $\bar{i}_{\bar{x}}: B H \rightarrow B G$ denote the corresponding closed immersions. (Observe that the fiber of $\bar{\pi}$ over any point $\bar{x}$ of $B \bar{G}$ is isomorphic to $B H$ and that the sheaves $R^{n} \bar{\pi}_{*}(K)$ are locally constant on $B \bar{G}$. Therefore we may in fact assume the point $\bar{x}$ is the base point $*$ of $B \bar{G}$.)

Let $L \bar{i}_{\bar{x}}^{*}=\bar{i}_{\bar{x}}^{-1}: D_{b}(B G) \rightarrow D_{b}(B H)$ and

$$
L i_{\bar{x}}^{*}=i_{\bar{x}}^{-1}: D_{+}\left(\operatorname{BiMod}\left(B \bar{G} ; R \bar{\pi}_{*}(\underline{\mathbb{Q}})\right)\right) \rightarrow D_{+}\left(\operatorname{BiMod}\left(\bar{x} ; R \bar{\pi}_{*}(\underline{\mathbb{Q}})_{\bar{x}}\right)\right)
$$

denote the obvious functors. Finally let $\bar{\pi}_{x}: B H \rightarrow \bar{x}$ denote the projection induced by $\bar{\pi}$. Now it suffices to show that the induced map $\bar{i}_{\bar{x}}^{-1}\left(L \bar{\pi}^{*}\left(R \bar{\pi}_{*}(K)\right) \rightarrow \bar{i}_{\bar{x}}^{-1}(K)\right.$ is a quasi-isomorphism. One may readily identify the above map to be: $L \bar{\pi}_{x}^{*}\left(i_{\bar{x}}^{-1}\left(R \bar{\pi}_{*}(K)\right) \rightarrow \bar{i}_{\bar{x}}^{-1}(K)\right.$. Now $i_{\bar{x}}^{-1}\left(R \bar{\pi}_{*}(K)\right)=\left(R \bar{\pi}_{*}(K)\right)_{\bar{x}}=$ $\mathbb{H}^{*}(B H ; K)$ by the proof of (i) of Theorem 1 while $\bar{i}_{\bar{x}}^{-1}(K)$ is the restriction of $K$ to $B H$, the geometric fiber of $\bar{\pi}$ at $\bar{x}$. In other words, we reduce to proving the theorem in case $G$ is replaced by $H$. In this case the naturality of the map in (i) shows that it suffices to prove it when $K$ is replaced by one of its cohomology sheaves. These cohomology sheaves are constant on $B H$ (since $H$ is connected and therefore $\mathrm{BH}$ simply connected); therefore (i) is clear in this case. Now (ii) follows immediately from (i).

In order to prove (iii), it suffices to show that if $P \in D_{+}^{\bar{G}}\left(\operatorname{BiMod}\left(B \bar{G} ; R \bar{\pi}_{*}(\underline{\mathbb{Q}})\right)\right)$, then $\operatorname{Hom}(P,-)$ applied to the above terms is an isomorphism. (Here Hom denotes the Hom in the derived category $D_{+}^{\bar{G}}\left(\operatorname{BiMod}\left(B \bar{G} ; R \bar{\pi}_{*}(\underline{\mathbb{Q}})\right)\right)$.) This follows readily in view of the adjunction between the $\mathcal{R} \mathcal{H o m}_{R \bar{\pi}_{*}(\underline{\mathbb{Q}})}$ and $\underset{R \bar{\pi}_{*}(\underline{\mathbb{Q}})}{\stackrel{L}{\otimes}}($ see $[\mathrm{K}-\mathrm{M}]$ part $\mathrm{V})$ and completes the proof.

(2.2.4) Given $M \in D_{+}^{\bar{G}}\left(\operatorname{BiMod}\left(B \bar{G} ; R \bar{\pi}_{*}(\underline{\mathbb{Q}})\right)\right)$, we define the dual of $M$ to be

$$
\mathcal{R H o m}_{R \bar{\pi}_{*}(\underline{\mathbb{Q}})}\left(M, R \bar{\pi}_{*}(\underline{\mathbb{Q}})\right) .
$$

This will be denoted $D_{R \bar{\pi}_{*}(\underline{\mathbb{Q}})}(M)$.

Now one may re-interpret $(2.2 .3)$ (iii) with $K^{\prime}=D(\underline{\mathbb{Q}}) \cong \underline{\mathbb{Q}}$ as

$$
D_{R \bar{\pi}_{*}(\underline{\mathbb{Q}})}\left(R \bar{\pi}_{*}(K)\right) \cong R \bar{\pi}_{*}(D(K))
$$


where $D(K)$ denotes the Verdier dual of $K$.

We may now complete the proof of statement (iv) in Theorem 1 as follows. Let $K \in D_{b}^{G}(X)$ be as in the hypothesis of Theorem 1 and let $L=R \pi_{G *}(K)$. One may now identify $\mathbb{H}^{n}(B H ; D(L))^{\bar{G}}$ with $H^{n}\left(R \Gamma\left(B \bar{G} ; R \bar{\pi}_{*} D(L)\right)\right) \cong H^{n}\left(R \Gamma\left(B \bar{G} ; D_{R \bar{\pi}_{*}(\mathbb{Q})}\left(R \bar{\pi}_{*}(L)\right)\right)\right.$ where the last isomorphism follows from (2.2.5). By definition, the last term equals $H^{n}\left(R \Gamma\left(B \bar{G} ; \mathcal{R} \mathcal{H} \operatorname{com}_{R \bar{\pi}_{*}(\mathbb{Q})}\left(R \bar{\pi}_{*}(L), R \bar{\pi}_{*}(\mathbb{Q})\right)\right.\right.$. Taking $X$ a point, $G$ the finite group $\bar{G}$ and $H$ the trivial subgroup, Theorem 1 (i) shows that the last term may be identified with $H^{n}\left(\mathcal{R} \mathcal{H} \operatorname{Hom}_{R \bar{\pi}_{*}(\mathbb{Q})}\left(R \bar{\pi}_{*}(L), R \bar{\pi}_{*}(\mathbb{Q})\right)\right)^{\bar{G}}$. Now the degeneration of the spectral sequence in $(2.2 .1)$ applies to identify this with $\left(\operatorname{Hom}_{H^{*}(B H ; \mathbb{Q})}^{n}\left(\mathbb{H}^{*}(B H ; L), H^{*}(B H ; \mathbb{Q})\right)\right)^{\bar{G}}$. Since $\mathbb{H}^{*}(B H ; L)=$ $\mathbb{H}^{*}\left(B H ; R \pi_{G *} K\right)=\mathbb{H}_{H}^{*}(X ; K)$ this completes the proof of Theorem 1.

\section{Filtration of global equivariant intersection cohomology by local equivariant intersection cohomology.}

We begin the proof of Theorem 2 with the following.

(3.1) Proposition. Let $X$ be a $G$-variety and let $\mathcal{O}$ be a closed $G$-orbit in $X$. Assume that $\mathcal{O}$ admits an attractive slice $\mathcal{S}$ at $x$, stable by a maximal torus $T_{x}$ of $G_{x}$. Now $\mathcal{O}^{T_{x}}$ is a union of connected components of $X^{T_{x}}$. Equivalently, $\mathcal{O}^{T_{x}}$ is open and closed in $X^{T_{x}}$.

Proof. The normalizer $N_{G}\left(T_{x}\right)$ acts on $X^{T_{x}}$ and on $\mathcal{O}^{T_{x}}$. Moreover, the quotient $N_{G}\left(T_{x}\right) / C_{G}\left(T_{x}\right)$ is a finite group.

It is well known that $\mathcal{O}^{T_{x}}=N_{G}\left(T_{x}\right) x$. (Indeed, let $g \in G$ such that $g x \in \mathcal{O}^{T_{x}}$. Then $T_{x}$ and $g^{-1} T_{x} g$ are maximal subtori of $G_{x}$. Thus, there exists $h \in G_{x}$ such that $g^{-1} T_{x} g=h T_{x} h^{-1}$. Now $g h \in N_{G}\left(T_{x}\right)$ and $g x=g h x \in N_{G}\left(T_{x}\right) x$.)

It follows that $\mathcal{O}^{T_{x}}$ is a finite union of closed orbits of $C_{G}\left(T_{x}\right)^{0}$. Thus, it suffices to check that $C_{G}\left(T_{x}\right)^{0} x$ is the connected component of $X^{T_{x}}$ through $x$. Because $C_{G}\left(T_{x}\right)^{0} x$ is smooth, it is enough to check that the Zariski tangent spaces of $C_{G}\left(T_{x}\right)^{0} x$ and of $X^{T_{x}}$ at $x$ coincide. For a scheme $Y$ and a point $y \in Y$, we denote by $\mathcal{T}_{y} Y$ the corresponding Zariski tangent space. Because the map $G \times \mathcal{S} \rightarrow X$ is smooth at $(e, x)$, its differential $\operatorname{Lie}(G) \times \mathcal{T}_{x} \mathcal{S} \rightarrow \mathcal{T}_{x} X$ is a surjective linear $T_{x}$-equivariant map. (Indeed, the differential of a smooth morphism is surjective, as follows e.g. from [A-K] IV (4.5) and V (1.8)). Moreover, because $\mathcal{S}$ is attractive, the fixed point set $\left(\mathcal{T}_{x} \mathcal{S}\right)^{T_{x}}$ consists of the origin. Thus, the natural map $\operatorname{Lie}(G)^{T_{x}} \rightarrow\left(\mathcal{T}_{x} X\right)^{T_{x}}$ is surjective. But $\operatorname{Lie}(G)^{T_{x}}=\operatorname{Lie}\left(\left(G^{T_{x}}\right)^{0}\right)$, and $\mathcal{T}_{x}\left(X^{T_{x}}\right)$ is contained in $\left(\mathcal{T}_{x} X\right)^{T_{x}}$. This proves our claim.

In the situation of (3.1), let $T$ denote a maximal torus of $G$ containing $T_{x}$ and let $W=N_{G}(T) / C_{G}(T)^{0}$, $W_{x}=N_{G_{x}}\left(T_{x}\right) / C_{G_{x}}\left(T_{x}\right)^{0}$ denote the corresponding Weyl groups. By (1.5.1), we may identify $H^{*}(B G)$ with $H^{*}(B T)^{W}$ and $H^{*}\left(B G_{x}\right)$ with $H^{*}\left(B T_{x}\right)^{W_{x}}$.

Let $\mathfrak{q}_{x}$ be the kernel of the restriction map $H^{*}(B T) \rightarrow H^{*}\left(B T_{x}\right)$; similarly, let $\mathfrak{p}_{\mathcal{O}}$ be the kernel of the restriction map $H^{*}(B G) \rightarrow H_{G}^{*}(\mathcal{O})=H^{*}\left(B G_{x}\right)$. Since the map $H^{*}\left(B G_{x}\right) \rightarrow H^{*}\left(B T_{x}\right)$ is injective and since $H^{*}(B G)$ identifies to $H^{*}(B T)^{W}$, this identifies $\mathfrak{p}_{\mathcal{O}}$ with the kernel of the restriction map $H^{*}(B T)^{W} \rightarrow H^{*}\left(B T_{x}\right)$. Therefore

$$
\mathfrak{p}_{\mathcal{O}}=\mathfrak{q}_{x} \cap H^{*}(B T)^{W}
$$

Let $\psi: \mathcal{O} \rightarrow X$ denote the inclusion. Let $\mathcal{L}$ denote a $G$-equivariant locally constant sheaf on an open $G$-stable smooth sub-variety of $X$.

(3.4) Proposition. In the above situation, the long exact sequence in $G$-equivariant hypercohomology

$$
\cdots \rightarrow \mathbb{H}_{G}^{*}\left(\mathcal{O} ; R \psi^{!} I C^{G}(X ; \mathcal{L})\right) \rightarrow \mathbb{H}_{G}^{*}\left(X ; I C^{G}(X ; \mathcal{L})\right) \rightarrow \mathbb{H}_{G}^{*}\left(X-\mathcal{O} ; I C^{G}(X ; \mathcal{L})\right) \rightarrow \cdots
$$

breaks up into split short exact sequences after localizing at the ideal $\mathfrak{p}_{\mathcal{O}}$. 
Proof. Recall that $H^{*}(B G)$ identifies with the subspace of $W$-invariants in $H^{*}(B T)$. More generally, $\mathbb{H}_{G}^{*}\left(X ; I C^{G}(\mathcal{L})\right)$ identifies with the subspace of $W$-invariants in $\mathbb{H}_{T}^{*}\left(X ; I C^{T}(\mathcal{L})\right)$, by $(1.5 .1)$. Thus, our statement means that the long exact sequence

$$
\cdots \rightarrow \mathbb{H}_{T}^{*}\left(\mathcal{O} ; R \psi^{!} I C^{T}(X ; \mathcal{L})\right)^{W} \rightarrow \mathbb{H}_{T}^{*}\left(X ; I C^{T}(X ; \mathcal{L})\right)^{W} \rightarrow \mathbb{H}_{T}^{*}\left(X-\mathcal{O} ; I C^{T}(X ; \mathcal{L})\right)^{W} \rightarrow \cdots
$$

breaks up into short exact sequences after inverting all elements of $H^{*}(B T)^{W}-\mathfrak{p}_{\mathcal{O}}$.

Let $\psi_{T_{x}}: \mathcal{O}^{T_{x}} \rightarrow X, \phi:(X-\mathcal{O})^{T_{x}} \rightarrow X$ and $\eta: X^{T_{x}} \rightarrow X$ denote the inclusion maps. By the localization theorem (1.5.4), we have the following isomorphisms:

$$
\begin{gathered}
\mathbb{H}_{T}^{*}\left(\mathcal{O}, R \psi^{!} I C^{T}(X ; \mathcal{L})_{\mathfrak{q}_{x}} \cong \mathbb{H}_{T}^{*}\left(\mathcal{O}^{T_{x}}, R \psi_{T_{x}}^{!} I C^{T}(X ; \mathcal{L})\right)_{\mathfrak{q}_{x}}\right. \\
\mathbb{H}_{T}^{*}\left(X-\mathcal{O} ; I C^{T}(X ; \mathcal{L})\right)_{\mathfrak{q}_{x}} \cong \mathbb{H}_{T}^{*}\left((X-\mathcal{O})^{T_{x}} ; R \phi^{!} I C^{T}(X ; \mathcal{L})\right)_{\mathfrak{q}_{x}}, \\
\mathbb{H}_{T}^{*}\left(X ; I C^{T}(X ; \mathcal{L})\right)_{\mathfrak{q}_{x}} \cong \mathbb{H}_{T}^{*}\left(X^{T_{x}} ; R \eta^{!} I C^{T}(X ; \mathcal{L})\right)_{\mathfrak{q}_{x}}
\end{gathered}
$$

Moreover, by (3.1), $X^{T_{x}}$ is the disjoint union of the closed subsets $(X-\mathcal{O})^{T_{x}}$ and $\mathcal{O}^{T_{x}}$. Thus, the $\operatorname{map} \mathbb{H}_{T}^{*}\left(\mathcal{O} ; R \psi^{!} I C^{T}(X ; \mathcal{L})\right) \rightarrow \mathbb{H}_{T}^{*}\left(X ; I C^{T}(X ; \mathcal{L})\right)$ is injective after localizing at $\mathfrak{q}_{x}$; in other words, the kernel $M$ of this map is killed by localizing at $\mathfrak{q}_{x}$. To conclude the proof, it is enough to check that the $H^{*}(B T)^{W}$-module $M^{W}$ is killed by localizing at $\mathfrak{p}_{\mathcal{O}}$.

Let $m \in M^{W}$. We can find $f \in H^{*}(B T)-\mathfrak{q}_{x}$ such that $f m=0$. Let $n$ be the cardinality of the orbit $W f$ and let $e_{1}(f), \ldots, e_{n}(f)$ be the elementary symmetric functions of the $w(f)(w \in W)$. Then each $e_{i}(f)$ is in $H^{*}(B T)^{W}$ and $e_{i}(f) m=0$. Moreover, since $f^{n}-e_{1}(f) f^{n-1}+\cdots+(-1)^{n} e_{n}(f)=0$, it follows that some $e_{i}(f)$ is not in $\mathfrak{p}_{\mathcal{O}}$ : otherwise, we would have $f^{n} \in \mathfrak{p}_{\mathcal{O}}$ and thus $f^{n} \in \mathfrak{q}_{x}$, a contradiction.

Remark. The results in (3.5) through (3.8) will show that the long exact sequence above breaks up into short exact sequences without localization at a prime ideal as in (3.4). Clearly this would prove Theorem $2(\mathrm{i})$.

(3.5) Proposition. Assume the hypotheses of Theorem 2 and denote by $i_{x}: x \rightarrow X$ the inclusion. Then we obtain isomorphisms

$$
\begin{aligned}
H^{*}\left(B C_{G_{x}}\left(T_{x}\right)^{0} ; i_{x}^{*} I C^{C_{G_{x}}\left(T_{x}\right)^{0}}(X ; \mathcal{L})\right) & \cong H^{*}\left(B T_{x} ; i_{x}^{*} I C^{T_{x}}(X ; \mathcal{L})\right) \\
& \cong H^{*}\left(B T_{x}\right) \otimes i_{x}^{*} \mathcal{H}^{*}(I C(X ; \mathcal{L})) \cong H^{*}\left(B C_{G_{x}}\left(T_{x}\right)^{0}\right) \otimes i_{x}^{*} \mathcal{H}^{*}(I C(X ; \mathcal{L}))
\end{aligned}
$$

These isomorphisms are equivariant for the natural action of $W_{x}$ on $H^{*}\left(B C_{G_{x}}\left(T_{x}\right)^{0} ; i_{x}^{*} I C^{C_{G_{x}}\left(T_{x}\right)^{0}}(X ; \mathcal{L})\right)$ and on $H^{*}\left(B C_{G_{x}}\left(T_{x}\right)^{0}\right)$, and for an induced action of $W_{x}$ on $i_{x}^{*} \mathcal{H}^{*}(I C(X ; \mathcal{L}))$.

Proof. The first and last isomorphisms are clear since $C_{G_{x}}\left(T_{x}\right)^{0} / T_{x}$ is a unipotent group. The hypotheses of Theorem 2 imply that $G^{0}$ has also only finitely many orbits on $X$ and therefore on the open dense $G$-stable subvariety $U_{1}$. Since $X$ is assumed to be equidimensional, all the connected components of $U_{1}$ are of the same dimension and each contains an open dense $G^{0}$-orbit. By our hypotheses, $U_{1}$ is the union of the corresponding $G$-orbits, all of which are also of the same dimension. Let $G x_{o}$ denote one of these orbits. Now the local system $\mathcal{L}$ corresponds to a representation of

$$
\pi_{1}\left(E G \times \underset{G}{\times} G x_{o}, x_{o}\right) \cong \pi_{1}\left(B G_{x_{o}}\right) \cong G_{x_{o}} / G_{x_{o}}^{0}
$$

Then one sees by (1.2.3) that $\mathcal{L}$ is semi-simple as a $G$-equivariant local system.

Let $\mathcal{S}$ be a slice at $x$. (See (0.2).) Recall that the map $G \times \mathcal{S} \rightarrow X$ is smooth; thus, the induced map $G \times \mathcal{S} \stackrel{\phi}{\rightarrow} X$ is smooth as well. Now the $G$-equivariant local system $\mathcal{L}$ pulls back to a $G$-equivariant

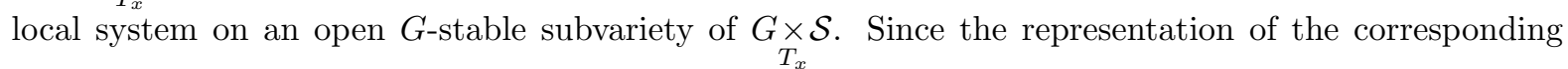
equivariant fundamental group is induced from the action of $\pi_{1}\left(E G \times G x_{o}, x_{o}\right)$ and this group is finite, 
this local system is also semi-simple as a $G$-equivariant local system. Under the equivalence of categories between $D_{b}^{G}\left(\underset{T_{x}}{\underset{S}{*}}\right.$ ) and $D_{b}^{T_{x}}(\mathcal{S})$, this corresponds to a semi-simple $T_{x}$ equivariant local system on an open $T_{x}$-stable subvariety, $\mathcal{S}_{o}$ of $\mathcal{S}-x$. We will denote this local system by $\mathcal{L}_{\mathcal{S}}$. Since $B T_{x}$ is simply connected, one may see that this local system restricted to each connected component of $\mathcal{S}_{o}$ is also semisimple (i.e. on forgetting the group action). Since the map $\phi$ is smooth, one observes that $\phi^{*}\left(I C^{G}(X ; \mathcal{L})\right)$ corresponds to $I C^{T_{x}}\left(S ; \mathcal{L}_{\mathcal{S}}\right)$ under the equivalence of categories between $D_{b}^{G}\left(G \times \underset{T_{x}}{\mathcal{S}}\right)$ and $D_{b}^{T_{x}}(\mathcal{S})$.

(3.5.1) In other words, if $j_{x}: x \rightarrow \mathcal{S}$ denotes the inclusion, one may observe that $i_{x}^{*} I C^{T_{x}}(X ; \mathcal{L}) \cong$ $j_{x}^{*} I C^{T_{x}}\left(\mathcal{S} ; \mathcal{L}_{\mathcal{S}}\right)$ in $D_{b}\left(B T_{x}\right)$.

Now consider the spectral sequence

$$
E_{2}^{s, t}=\mathbb{H}^{s}\left(B T_{x} ; \mathcal{H}^{t}\left(i_{x}^{*} I C^{T_{x}}(X ; \mathcal{L})\right)\right) \Rightarrow \mathbb{H}^{s+t}\left(B T_{x} ; i_{x}^{*}\left(I C^{T_{x}}(X ; \mathcal{L})\right)\right)
$$

We will first prove that this spectral sequence degenerates. For this, one may invoke (1.7.2) (iii) to reduce to the case where $\mathcal{L}$ is the constant local system (specifically, one first normalizes $X$, obtaining a disjoint union of irreducible normal varieties $\tilde{X}_{i}$. Then one normalizes each $\tilde{X}_{i}$ in the function field of $G / G_{\tilde{x}_{i}}^{0}$, where $G \tilde{x}_{i}$ is open in $\tilde{X}_{i}$.) Now (3.5.1) shows that we may replace $X$ by $\mathcal{S}$ and the equivariant intersection cohomology complex $I C^{T_{x}}(X ; \mathcal{L})$ on $X$ by the corresponding complex $I C^{T_{x}}\left(\mathcal{S} ; \mathcal{L}_{\mathcal{S}}\right)$ on $\mathcal{S}$. Therefore, in (3.5.2) through (3.5.10), we will replace $X$ by $\mathcal{S}$, and assume that $\mathcal{L}$ is constant.

Next, let $\mathcal{T}_{x} \mathcal{S}$ be the tangent space to $\mathcal{S}$ at $x$; then there exists a $T_{x}$-equivariant closed immersion $i: \mathcal{S} \rightarrow \mathcal{T}_{x} \mathcal{S}$. Moreover, there exists an injective one parameter subgroup $\lambda: \mathbb{G}_{m} \rightarrow T_{x}$ such that the action of $\mathbb{G}_{m}$ on $\mathcal{T}_{x} \mathcal{S}$ through $\lambda$ has only positive weights. (See (0.2) and (A.1) for the definition and basic properties of slices.) For simplicity, denote $\lambda\left(\mathbb{G}_{m}\right)$ by $T^{\prime}$. Now the quotients $Y=(\mathcal{S}-x) / T^{\prime}$ and $\mathbb{P}=\left(\mathcal{T}_{x} \mathcal{S}-0\right) / T^{\prime}$ exist and the latter is a weighted projective space containing the former as a closed subvariety. Moreover $Y$ is also equidimensional. Further, the map $\pi: \mathcal{S}-x \rightarrow Y$ is the restriction of the map $\bar{\pi}: \mathcal{T}_{x} \mathcal{S}-0 \rightarrow \mathbb{P}$, both with fibers isomorphic to $\mathbb{G}_{m}$. (We will refer to both these maps as $\mathbb{G}_{m}$-quasi-fibrations, for want of a better term.) The first Chern class of the $\mathbb{G}_{m}$-quasi-fibration $\bar{\pi}$ exists as the class in $H^{2}(\mathbb{P})$ of a hypersurface. To see this, observe that $\mathbb{P}$ is the Proj of a finitely generated graded algebra and therefore there exists a positive integer $n_{0}$ such that, for any positive integer $n$, the sheaf $\mathcal{O}_{\mathbb{P}}\left(n n_{0}\right)$ is invertible and very ample. (See [Dolg].) Let $i: \mathbb{P} \rightarrow \mathbb{P}^{N}$ be the closed immersion defined by $\mathcal{O}_{\mathbb{P}}\left(n_{0}\right)$, where $\mathbb{P}^{N}$ denotes the usual projective space of dimension $N$ over $k$. Then the Hard Lefschetz theorem (as in [B-B-D] Théorème (5.4.10)) applies to the class of a hyperplane in $\mathbb{P}$. We will denote this class by $c_{1}(\pi)$.

Observe that $T_{x}$ acts on $Y$ through the action of $T_{x} / T^{\prime}$ and the map $\pi: \mathcal{S}-x \rightarrow Y$ is $T_{x}$-equivariant. Clearly the constant $T_{x}$-equivariant local system $\underline{\mathbb{Q}}_{\mathcal{S}}$ on an open $T_{x}$-stable subvariety of $\mathcal{S}-x$ descends to the constant $T_{x}$-equivariant local system $\mathbb{Q}_{Y}$ on an open $T_{x}$-stable subvariety $Y_{o}$ of $Y$. Let $Y_{o}(i)$, $i=1, \ldots, n$ denote the connected components of $Y_{o}$. (Observe that these are stable by the action of $T_{x}$ and of the same dimension.) We define $\left(T_{x}\right.$-equivariant) local systems $\mathbb{Q}_{Y}(i)$ on $Y_{o}$ by $\underline{\mathbb{Q}}_{Y}(i) \mid Y_{o}(j)$ equals $\underline{\mathbb{Q}}_{Y} \mid Y_{o}(j)$ if $i=j$, and is trivial otherwise. Now $\underline{\mathbb{Q}}_{Y}$ is the sum of the local systems $\underline{\mathbb{Q}}_{Y}(i)$ each of which is a simple local system on $Y_{o}$.

If $I C^{T_{x}}(Y)\left(I C^{T_{x}}(\mathcal{S}-x)\right)$ denotes the equivariant intersection cohomology complex on $Y$ with respect to $\underline{\mathbb{Q}}_{Y}$ (on $\mathcal{S}-x$ with respect to $\underline{\mathbb{Q}}_{\mathcal{S}}$, respectively ), then

$$
\mathcal{H}_{\text {perv }}^{n}\left(R \pi_{*}\left(I C^{T_{x}}(\mathcal{S}-x)\right)\right)=I C^{T_{x}}(Y) \text { if } n=0,1 \text { and is trivial otherwise. }
$$

This follows from (A.3) in the appendix; here $\mathcal{H}_{\text {perv }}^{n}$ denotes the $n$-th perverse cohomology. Moreover, $I C^{T_{x}}(Y) \cong \oplus_{i} I C^{T_{x}}\left(Y ; \underline{\mathbb{Q}}_{Y}(i)\right), I C(Y) \cong \oplus_{i} I C\left(Y ; \mathbb{Q}_{Y}(i)\right)$ and each $I C\left(Y ; \mathbb{Q}_{Y}(i)\right)$ is a mixed simple perverse sheaf (or rather a simple perverse sheaf of geometric origin, in characteristic 0 ) on $Y$ and therefore the corresponding perverse sheaf defined over a finite field is pure. (See (0.5), [B-B-D] p. 136 and p. 163. The hypothesis (0.5) shows that, in positive characteristics, the above intersection cohomology complex (with $\mathbb{Q}$ replaced by $\mathbb{Q}_{l}$ ) is obtained from the corresponding one defined over a 
finite field. Therefore the arguments in [B-B-D] p. 136 apply in this case. In characteristic 0, the arguments in [B-B-D] p. 163 enable one to reduce to the case of varieties defined over finite fields.) It follows that $I C(Y)$ is also pure.

One now obtains the following commutative diagram:

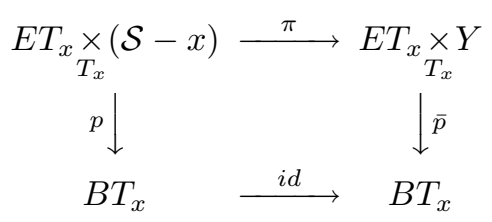

If $F \in D_{b}^{T_{x}}(\mathcal{S}-x), R p_{*} F \in D_{b}\left(B T_{x}\right)$ will denote the corresponding complex. Next we show that there exists a spectral sequence:

$$
E_{2}^{s, t}=R^{s} \bar{p}_{*}\left(\mathcal{H}_{\text {perv }}^{t}\left(R \pi_{*}\left(I C^{T_{x}}(\mathcal{S}-x)\right)\right)\right) \Rightarrow \mathcal{H}^{s+t}\left(R p_{*}\left(I C^{T_{x}}(\mathcal{S}-x)\right)\right)
$$

For this we use the perverse $t$-structure to obtain a distinguished triangle:

$$
\begin{aligned}
\tau_{\leq t-1}^{p e r v}\left(R \pi_{*}\left(I C^{T_{x}}(\mathcal{S}-x)\right)\right) & \rightarrow \tau_{\leq t}^{\text {perv }}\left(R \pi_{*}\left(I C^{T_{x}}(\mathcal{S}-x)\right)\right) \\
& \rightarrow \mathcal{H}_{\text {perv }}^{t}\left(R \pi_{*}\left(I C^{T_{x}}(\mathcal{S}-x)\right)\right)[-t]
\end{aligned}
$$

The above spectral sequence is obtained by applying $R \bar{p}_{*}$ to the above distinguished triangle. Since $E_{2}^{s, t}=0$ for $t$ different from 0 or 1 (see (A.3)) we obtain a long exact sequence:

$$
\begin{aligned}
& \cdots \rightarrow R^{n-2} \bar{p}_{*}\left(\mathcal{H}_{\text {perv }}^{1}\left(R \pi_{*}\left(I C^{T_{x}}(\mathcal{S}-x)\right)\right)\right)(-1) \stackrel{\cup c_{1}(\pi)}{\longrightarrow} R^{n} \bar{p}_{*}\left(\mathcal{H}_{\text {perv }}^{0}\left(R \pi_{*}\left(I C^{T_{x}}(\mathcal{S}-x)\right)\right)\right) \\
& \rightarrow \mathcal{H}^{n}\left(R \bar{p}_{*}\left(R \pi_{*}\left(I C^{T_{x}}(\mathcal{S}-x)\right)\right)\right) \rightarrow R^{n-1} \bar{p}_{*}\left(\mathcal{H}_{p e r v}^{1}\left(R \pi_{*}\left(I C^{T_{x}}(\mathcal{S}-x)\right)\right)\right)(-1) \\
& \stackrel{\cup c_{1}(\pi)}{\longrightarrow} R^{n+1} \bar{p}_{*}\left(\mathcal{H}_{\text {perv }}^{0}\left(R \pi_{*}\left(I C^{T_{x}}(\mathcal{S}-x)\right)\right)\right) \rightarrow \cdots
\end{aligned}
$$

Moreover, for any geometric point $\xi$ of $B T_{x}$ and for any integer $m$, we have the identifications:

$$
\begin{gathered}
R^{m} \bar{p}_{*}\left(\mathcal{H}_{\text {perv }}^{n}\left(R \pi_{*}\left(I C^{T_{x}}(\mathcal{S}-x)\right)\right)\right)_{\xi} \cong R^{m} \bar{p}_{*}\left(I C^{T_{x}}(Y)\right)_{\xi} \cong I H^{m}(Y) \quad(n=0,1), \\
R^{m} p_{*}\left(I C^{T_{x}}(\mathcal{S}-x)\right)_{\xi} \cong I H^{m}(\mathcal{S}-x) .
\end{gathered}
$$

The first follows from (A.3) and the second is clear. Therefore, on taking stalks at $\xi$, the long exact sequence in (3.5.4) provides the Wang exact sequence (see [K-L] p. 194):

$$
\begin{aligned}
\cdots \rightarrow I H^{n-2}(Y)(-1) \stackrel{\cup c_{1}(\pi)}{\longrightarrow} I H^{n}(Y) \rightarrow I H^{n}(\mathcal{S}-x) & \\
& \rightarrow I H^{n-1}(Y)(-1) \stackrel{\cup c_{1}(\pi)}{\longrightarrow} I H^{n+1}(Y) \rightarrow \cdots
\end{aligned}
$$

The Hard Lefschetz theorem in intersection cohomology shows that the first and last maps are injective for all $n \leq \operatorname{dim} Y=\operatorname{dim} \mathcal{S}-1$. Therefore, the long exact sequence in (3.5.4) breaks up into short exact sequences for all $n \leq \operatorname{dim} Y$. In particular it shows that for all $n \leq \operatorname{dim} Y$,

$$
R^{n} p_{*}\left(I C^{T_{x}}(\mathcal{S}-x)\right)=\mathcal{H}^{n}\left(R \bar{p}_{*}\left(R \pi_{*}\left(I C^{T_{x}}(\mathcal{S}-x)\right)\right)\right)
$$

is a complement to the image of the map $\cup c_{1}(\pi)$ i.e. in the above range, $R^{n} p_{*}\left(I C^{T_{x}}(\mathcal{S}-x)\right)$ is the primitive part of $R^{n} \bar{p}_{*}\left(I C^{T_{x}}(Y)\right)$ for the action of $c_{1}(\pi)$.

Observe further that $\mathcal{H}^{n}\left(I C^{T_{x}}(\mathcal{S})\right)_{x}=0$ for all $n>\operatorname{dim} Y$. (This follows from the axioms of intersection cohomology, see (1.4.4)(ii).) One may observe exactly as in [K-L] p. 194, that one obtains the isomorphism for all (geometric) points $\xi \in B T_{x}$ :

$$
R^{n} p_{*}\left(I C^{T_{x}}(\mathcal{S}-x)\right)_{\xi} \cong \underset{I}{I} H^{n}(\mathcal{S}-x) \cong I H^{n}(\mathcal{S}) \cong \mathcal{H}^{n}(I C(\mathcal{S}))_{x}
$$


for all $n \leq \operatorname{dim} Y$.

Next recall that $x$ is a fixed point for the $T_{x}$-action on $X$. Therefore $i_{x}^{*}\left(I C^{T_{x}}(\mathcal{S})\right)$ is a complex in $D_{b}\left(B T_{x}\right)$ whose cohomology sheaves $\mathcal{H}^{n}\left(i_{x}^{*}\left(I C^{T_{x}}(\mathcal{S})\right)\right)$ are constant on $B T_{x}$. Similarly $R p_{*} I C^{T_{x}}(\mathcal{S}-x)$ and $R \bar{p}_{*} I C^{T_{x}}(Y)$ belong to $D_{b}\left(B T_{x}\right)$. The constant sheaf on $B T_{x}$ with stalks isomorphic to $\mathcal{H}^{n}\left(i_{x}^{*} I C(\mathcal{S})\right)$ is $\mathcal{H}^{n}\left(I C^{T_{x}}(\mathcal{S})\right)_{x}$. Therefore, combining (3.5.7) and (3.5.8), we obtain the identification:

$\mathcal{H}^{n}\left(i_{x}^{*} I C^{T_{x}}(\mathcal{S})\right)$ is the primitive part of $R^{n} \bar{p}_{*}\left(I C^{T_{x}}(Y)\right)$.

Now consider the cohomological functor $F \rightarrow H^{*}\left(B T_{x} ; F\right), D_{b}\left(B T_{x}\right) \rightarrow$ (abelian groups) obtained by taking the cohomology of $B T_{x}$ with respect to the complex $F$. According to [De] Theorem (1.5) (see (1.5.2)), the spectral sequence:

$$
E_{2}^{s, t}=H^{*}\left(B T_{x} ; \mathcal{H}^{t}(F)[s]\right) \Rightarrow H^{*}\left(B T_{x} ; F[s+t]\right)
$$

degenerates when $F=R \bar{p}_{*} I C^{T_{x}}(Y)$. Moreover the differentials are all trivial. Therefore one may conclude that the differentials in the corresponding spectral sequence for $F=i_{x}^{*}\left(I C^{T_{x}}(\mathcal{S})\right)$ are also trivial and that it degenerates. Thus, one obtains the identification:

$$
H^{*}\left(B T_{x} ; i_{x}^{*}\left(I C^{T_{x}}(\mathcal{S})\right)\right) \cong H^{*}\left(B T_{x}\right) \otimes i_{x}^{*} \mathcal{H}^{*}(I C(\mathcal{S}))
$$

In view of (1.7.2) (iii) the last identification holds with the local system $\mathcal{L}_{\mathcal{S}}$ in the place of the constant local system. Now (3.5.1) shows that the same isomorphism holds and the spectral sequence in (3.5.9) degenerates where $X$ is the original variety and with the local system $\mathcal{L}$ in the place of $\mathcal{L}_{\mathcal{S}}$.

It follows from Theorem 1 that there exists an action of the Weyl group $W_{x}$ on $H^{*}\left(B T_{x} ; K\right)$ for any $K \in D_{b}^{N_{G}\left(T_{x}\right)}(p t)$ and that this action is natural in $K$. Therefore there exists an action of $W_{x}$ on $H^{*}\left(B T_{x} ; \tau_{\leq m}\left(I C^{T_{x}}(X ; \mathcal{L})\right)_{x}\right)$ that is natural in $m$. It follows that the $W_{x}$-action is compatible with the differentials of the spectral sequence in (3.5.9). Since the above spectral sequence degenerates, the isomorphism of the abutment with $\underset{r, s}{\oplus} E_{2}^{s, t}$ is one of $W_{x}$-modules.

To complete the proof of Proposition (3.5), we must show that there exists an action of $W_{x}$ on $i_{x}^{*} \mathcal{H}^{*}(I C(X ; \mathcal{L}))$ such that the second and third isomorphism of this proposition are equivariant. But this results from the following standard result below with $A=H^{*}\left(B T_{x}\right), M=H^{*}\left(B T_{x} ; \quad i_{x}^{*} \mathcal{H}^{*}\left(I C^{T_{x}}(X ; \quad \mathcal{L})\right)\right)$ and $V=i_{x}^{*} \mathcal{H}^{*}(I C(X ; \quad \mathcal{L}))$.

(3.6) Lemma. Let $A$ be a positively graded $k$-algebra with an action of a finite group $F$ and let $M$ be a positively graded $A$-module endowed with a compatible action of $F$. If the $A$-module $M$ is free, then there exists a graded $F$-stable subspace $V$ of $M$ such that the natural map $A \otimes V \rightarrow M$ is an isomorphism.

Proof. Let $A^{+}$be the maximal graded ideal of $A$. Consider the surjective, $F$-equivariant map $p: M \rightarrow$ $M / A^{+} M$. Since $F$ is a finite group, there exists a graded $F$-stable subspace $V$ of $M$ which is mapped isomorphically to $M / A^{+} M$ by $p$. Then $A \otimes V \rightarrow M$ is an isomorphism, by the graded Nakayama lemma.

Remarks. (i) Observe that exactly the same proof holds in positive characteristics by replacing the constant sheaf $\underline{\mathbb{Q}}$ with $\underline{\mathbb{Q}}_{l}$.

(ii) The existence of the Wang exact sequence as in (3.5.6) is stated in [K-L] p. 194 without proofs.

(3.7) Corollary. Assume the hypotheses of Theorem 2. Then we obtain the isomorphisms:

(i) $\mathbb{H}^{*}\left(B N_{G_{x}}\left(T_{x}\right) ; R i_{x}^{!} I C^{N_{G_{x}}\left(T_{x}\right)}(X ; \mathcal{L})\right) \cong \mathbb{H}^{*}\left(B N_{G_{x}}\left(T_{x}\right) ; \mathcal{H}^{*}\left(R i_{x}^{!} I C^{N_{G_{x}}\left(T_{x}\right)}(X ; \mathcal{L})\right)\right)$

$$
\cong\left(\mathbb{H}^{*}\left(B C_{G_{x}}\left(T_{x}\right)^{0} ; \mathcal{H}^{*}\left(R i_{x}^{!} I C^{C_{G_{x}}\left(T_{x}\right)^{0}}(X ; \mathcal{L})\right)\right)\right)^{W_{x}} \cong\left(\mathbb{H}^{*}\left(B T_{x} ; \mathcal{H}^{*}\left(R i_{x}^{!} I C^{T_{x}}(X ; \mathcal{L})\right)\right)\right)^{W_{x}}
$$

and

$$
\mathbb{H}^{*}\left(B T_{x} ; R i_{x}^{!} I C^{T_{x}}(X ; \mathcal{L})\right) \cong H^{*}\left(B T_{x}\right) \otimes \mathcal{H}^{*}\left(R i_{x}^{!} I C(X ; \mathcal{L})\right)
$$


Proof. Recall that

$$
\begin{aligned}
R i_{x}^{!} I C^{N_{G_{x}}\left(T_{x}\right)}(X ; \mathcal{L}) \cong & D i_{x}^{*} D\left(I C^{N_{G_{x}}\left(T_{x}\right)}(X ; \mathcal{L})\right) \cong D i_{x}^{*}\left(I C^{N_{G_{x}}\left(T_{x}\right)}\left(X ; \mathcal{L}^{\vee}\right)[2 d]\right) \\
& \cong D i_{x}^{*}\left(I C^{N_{G_{x}}\left(T_{x}\right)}\left(X ; \mathcal{L}^{\vee}\right)\right)[-2 d]
\end{aligned}
$$

where $d$ is the dimension of $X$. We apply Theorem 1 (iv) with $K=i_{x}^{*} I C^{N_{G_{x}}\left(T_{x}\right)}\left(X ; \mathcal{L}^{\vee}\right), G=N_{G_{x}}\left(T_{x}\right)$ and $H=C_{G_{x}}\left(T_{x}\right)^{\circ}$. It follows by (3.5) that the hypothesis that $H^{*}\left(B C_{G_{x}}^{0}\left(T_{x}\right) ; K\right)$ be a free module over $H^{*}\left(B C_{G_{x}}^{0}\left(T_{x}\right)\right)$ is satisfied. Next recall that $C_{G_{x}}^{0}\left(T_{x}\right) / T_{x}$ is unipotent. Therefore one obtains the isomorphism

$$
\left.\mathbb{H}^{*}\left(B C_{G_{x}}^{0}\left(T_{x}\right) ; K\right) \cong \mathbb{H}^{*}\left(B T_{x} ; K\right)\right) .
$$

Thus:

(3.7.1) $H^{*}\left(B N_{G_{x}}\left(T_{x}\right) ; R i_{x}^{!} I C^{N_{G_{x}}\left(T_{x}\right)}(X ; \mathcal{L})\right)=H^{*}\left(B N_{G_{x}}\left(T_{x}\right) ; D\left(i_{x}^{*} I C^{N_{G_{x}}\left(T_{x}\right)}\left(X ; \mathcal{L}^{\vee}\right)[2 d]\right)\right)$

$$
\begin{aligned}
\cong\left(\operatorname{Hom}_{\left.H^{*}\left(B T_{x}\right)\right)}\right. & \left.\left(\mathbb{H}^{*}\left(B T_{x} ; i_{x}^{*} I C^{T_{x}}\left(X ; \mathcal{L}^{\vee}\right)[2 d]\right)\right) ; H^{*}\left(B\left(T_{x}\right)\right)\right)^{W_{x}} \\
& \cong\left(\operatorname{Hom}_{\left.H^{*}\left(B T_{x}\right)\right)}\left(\mathbb{H}^{*}\left(B T_{x} ; \mathcal{H}^{*}\left(i_{x}^{*} I C^{T_{x}}\left(X ; \mathcal{L}^{\vee}\right)[2 d]\right)\right) ; H^{*}\left(B T_{x}\right)\right)\right)^{W_{x}}
\end{aligned}
$$

Observe that the dualizing sheaf on $D_{+}^{N_{G_{x}}\left(T_{x}\right)}(x ; \mathbb{Q})$ is in fact the constant sheaf $\mathbb{Q}$. Moreover the complex $i_{x}^{*} I C^{N_{x} G\left(T_{x}\right)}\left(X ; \mathcal{L}^{\vee}\right)$ has locally constant cohomology sheaves on $B N_{G}\left(T_{x}\right)$. Therefore one may readily obtain the quasi-isomorphism

$$
D\left(\mathcal{H}^{*}\left(i_{x}^{*} I C^{N_{G_{x}}\left(T_{x}\right)}\left(X ; \mathcal{L}^{\vee}\right)\right)\right)[-2 d] \cong \mathcal{H}^{*}\left(D\left(i_{x}^{*} I C^{N_{G_{x}}\left(T_{x}\right)}\left(X ; \mathcal{L}^{\vee}\right)[2 d]\right)\right) \cong \mathcal{H}^{*}\left(R i_{x}^{!} I C^{N_{G_{x}}\left(T_{x}\right)}(X ; \mathcal{L})\right) .
$$

Now we apply Theorem 1 (iv) with $G=N_{G_{x}}\left(T_{x}\right), H=C G_{x} G\left(T_{x}\right)^{o}$ and $K=\mathcal{H}^{*}\left(i_{x}^{*} I C^{N_{G_{x}}\left(T_{x}\right)}\left(X ; \mathcal{L}^{\vee}\right)\right)[2 d]$ (observe that this applies here since

$$
\left.\mathbb{H}^{*}\left(B C_{G_{x}}^{0}\left(T_{x}\right) ; \mathcal{H}^{*}\left(i_{x}^{*} I C^{T_{x}}\left(X ; \mathcal{L}^{\vee}\right)\right)\right) \cong H^{*}\left(B C_{G_{x}}^{0}\left(T_{x}\right)\right) \otimes \mathcal{H}^{*}\left(i_{x}^{*} I C^{T_{x}}\left(X ; \mathcal{L}^{\vee}\right)\right)\right)
$$

to obtain:

(3.7.2) $H^{*}\left(B N_{G_{x}}\left(T_{x}\right) ; \mathcal{H}^{*}\left(D\left(i_{x}^{*} I C^{N_{G_{x}}\left(T_{x}\right)}\left(X ; \mathcal{L}^{\vee}\right)\right)[2 d]\right)\right) \cong H^{*}\left(B N_{G_{x}}\left(T_{x}\right) ; D\left(\mathcal{H}^{*}\left(i_{x}^{*} I C^{N_{G_{x}}\left(T_{x}\right)}\left(X ; \mathcal{L}^{\vee}\right)\right)[2 d]\right)\right)$

$$
\cong\left(\operatorname{Hom}_{H^{*}\left(B T_{x}\right)}\left(\mathbb{H}^{*}\left(B T_{x} ; \mathcal{H}^{*}\left(i_{x}^{*} I C^{T_{x}}\left(X ; \mathcal{L}^{\vee}\right)[2 d]\right)\right) ; H^{*}\left(B T_{x}\right)\right)\right)^{W_{x}}
$$

Since the terms in (3.7.1) and (3.7.2) are the same we obtain the isomorphism in (i). The proof of (ii) is similar and is skipped.

Now we are in a position to complete the proof of Theorem 2.

(3.8) Proof of Theorem 2. Let $\mathcal{O}$ be a closed $G$-orbit in $X$ with inclusion map $\psi: \mathcal{O} \rightarrow X$ and let $\mathcal{L}$ denote a $G$-equivariant local system on the union of all open $G$-orbits. Consider the long exact sequence of $H^{*}(B G)$-modules:

$$
\cdots \rightarrow \mathbb{H}_{G}^{*}\left(\mathcal{O} ; R \psi^{!} I C^{G}(X ; \mathcal{L})\right) \rightarrow \mathbb{H}_{G}^{*}\left(X ; I C^{G}(X ; \mathcal{L})\right) \rightarrow \mathbb{H}_{G}^{*}\left(X-\mathcal{O} ; I C^{G}(X ; \mathcal{L})\right) \rightarrow \cdots
$$

We will show that this sequence splits into short exact sequences and that the $H^{*}(B G)$-module $\mathbb{H}_{G}^{*}\left(\mathcal{O} ; R \psi^{!} I C^{G}(X ; \mathcal{L})\right)$ is isomorphic to $\left(H^{*+2} \operatorname{dim}(\mathcal{O})\left(B T_{x} ; \mathcal{H}^{*}\left(R i_{x}^{!} I C(X ; \mathcal{L})\right)\right)\right)^{W_{x}}$ for $x \in \mathcal{O}$. Then our assertion will follow by induction on the number of orbits.

Next we observe that

$$
j_{x}^{*} R \psi^{!} I C^{G}(X ; \mathcal{L}) \cong R i_{x}^{!} I C^{G_{x}}(X ; \mathcal{L})[2 \operatorname{dim}(\mathcal{O})]
$$

where $i_{x}: B G_{x} \cong E G_{x} \underset{G_{x}}{\times x} \rightarrow \underset{G}{E} \underset{G}{\times}$ and $j_{x}: E G_{x} \underset{G_{x}}{\times x} \rightarrow \underset{G}{E} \underset{G}{\times}\left(G / G_{x}\right)$ denote the obvious inclusions.

(To see this recall the equivalence of derived categories:

$$
D^{G_{x}}(x) \stackrel{p_{1}^{*}}{\simeq} D^{G \times G_{x}}(G \times x) \stackrel{p_{2}^{*}}{\simeq} D^{G}\left(G / G_{x}\right)
$$


where $p_{1}: G \times x \rightarrow x$ and $p_{2}: G \times x \rightarrow G / G_{x}$ are the obvious maps. Then the complex in $D^{G_{x}}(x)$ that corresponds to $R \psi^{!} I C^{G}(X ; \mathcal{L})$ is $j_{x}^{*}\left(R \psi^{!} I C^{G}(X ; \mathcal{L})\right)$. (Use the fact the map $p_{1}$ has a (non-equivariant) section.) Next observe that $j_{x}$ is a regular closed immersion of codimension $\operatorname{dim}(\mathcal{O})$ and the cohomology sheaves of $R \psi^{!}\left(I C^{G}(X ; \mathcal{L})\right)$ are $G$-equivariant and hence locally constant on $\mathcal{O}$. Therefore $j_{x}^{*}\left(R \psi^{!} I C^{G}(X ; \mathcal{L})\right)$ identifies canonically with $R i_{x}^{!} I C^{G_{x}}(X ; \mathcal{L})[2 \operatorname{dim}(\mathcal{O})]$. See also (1.4.4).)

Therefore (see (1.6.2) with $H=G_{x}$ ) one obtains the isomorphism:

$$
\mathbb{H}_{G}^{*}\left(\mathcal{O} ; R \psi^{!} I C^{G}(X ; \mathcal{L})\right) \cong \mathbb{H}^{*+2 \operatorname{dim}(\mathcal{O})}\left(B G_{x} ; R i_{x}^{!} I C^{G_{x}}(X ; \mathcal{L})\right) .
$$

By (1.5.1)(ii) one may identify $\mathbb{H}^{*}\left(B G_{x} ; R i_{x}^{!} I C^{G_{x}}(X ; \mathcal{L})\right)$ with

$$
\mathbb{H}^{*}\left(B N_{G}\left(T_{x}\right) ; R i_{x}^{!} I C^{N_{G}\left(T_{x}\right)}(X ; \mathcal{L})\right) \cong \mathbb{H}^{*}\left(B T_{x} ; R i_{x}^{!} I C^{T_{x}}(X ; \mathcal{L})\right)^{W_{x}} .
$$

Moreover (3.7) shows that one may identify the latter with

$$
\mathbb{H}^{*}\left(B T_{x} ; \mathcal{H}^{*}\left(R i_{x}^{!} I C^{T_{x}}(X ; \mathcal{L})\right)\right)^{W_{x}} \cong\left(H^{*}\left(B T_{x}\right) \otimes I H_{x}^{*}(X ; \mathcal{L})\right)^{W_{x}} .
$$

i.e. we obtain the identification:

$$
I H_{\mathcal{O}, G}^{*}(X ; \mathcal{L})=\mathbb{H}_{G}^{*}\left(\mathcal{O} ; R \psi^{!} I C^{G}(X ; \mathcal{L})\right) \cong\left(H^{*+2} \operatorname{dim}(\mathcal{O})\left(B T_{x}\right) \otimes I H_{x}^{*}(X ; \mathcal{L})\right)^{W_{x}} .
$$

In order to prove the first statement of the theorem, observe that it suffices to show that the map

$$
\mathbb{H}_{G}^{*}\left(\mathcal{O} ; R \psi^{!} I C^{G}(X ; \mathcal{L})\right) \rightarrow \mathbb{H}_{G}^{*}\left(X ; I C^{G}(X ; \mathcal{L})\right)
$$

is injective. For this, observe that, by (3.4), the map

$$
\mathbb{H}_{G}^{*}\left(\mathcal{O} ; R \psi^{!} I C^{G}(X ; \mathcal{L})\right)_{\mathfrak{p}_{\mathcal{O}}} \rightarrow \mathbb{H}_{G}^{*}\left(X ; I C^{G}(X ; \mathcal{L})\right)_{\mathfrak{p}_{\mathcal{O}}}
$$

is injective. Now the commutative square

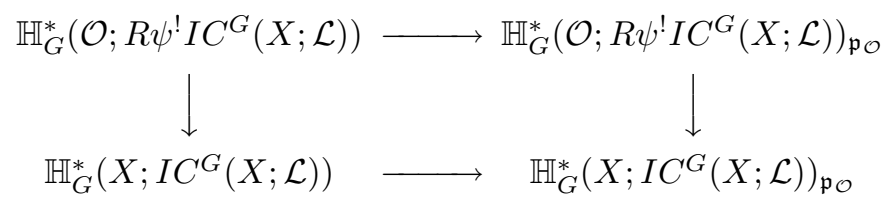

shows that it suffices to check injectivity of the map

$$
\mathbb{H}_{G}^{*}\left(\mathcal{O} ; R \psi^{!} I C^{G}(X ; \mathcal{L})\right) \rightarrow \mathbb{H}_{G}^{*}\left(\mathcal{O} ; R \psi^{!} I C^{G}(X ; \mathcal{L})\right)_{\mathfrak{p}_{\mathcal{O}}} .
$$

In view of the above observations, this map may be identified with the map

$$
\mathbb{H}^{*+2 \operatorname{dim}(\mathcal{O})}\left(B T_{x} ; \mathcal{H}^{*}\left(R i_{x}^{!} I C^{T_{x}}(X ; \mathcal{L})\right)\right)^{W_{x}} \rightarrow \mathbb{H}^{*+2 \operatorname{dim}(\mathcal{O})}\left(B T_{x} ; \mathcal{H}^{*}\left(R i_{x}^{!} I C^{T_{x}}(X ; \mathcal{L})\right)\right)_{\mathfrak{p}_{\mathcal{O}}}^{W_{x}}
$$

Now the commutative square

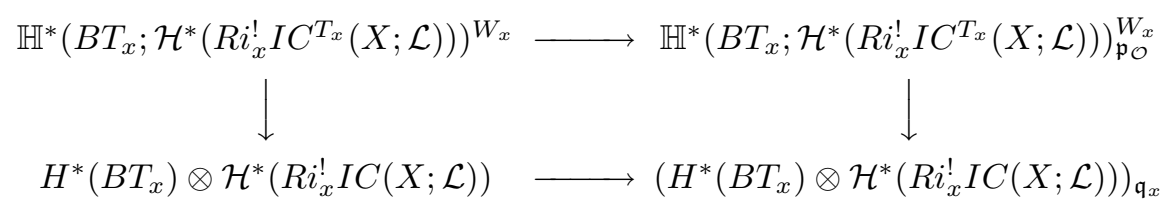

shows that the top row is indeed injective. (Observe that the right vertical map exists since $H^{*}(B G)-p_{\mathcal{O}}$ is contained in $H^{*}(B T)-\mathfrak{q}_{x}$. The left vertical map and the bottom map are clearly injective. Therefore so is the top horizontal map.) 
It remains to obtain the second statement of Theorem 2. Let $W_{x}^{0}$ denote $N_{G_{x}^{0}}\left(T_{x}\right) / C_{G_{x}^{0}}\left(T_{x}\right)$. Now $W_{x}^{0}$ is the kernel of the obvious map $W_{x} \rightarrow G_{x} / G_{x}^{0}$. This map is surjective: indeed, if $g \in G_{x}$, then $g T_{x} g^{-1}$ is a maximal torus of $G_{x}^{0}$. Thus, changing $g$ in its $G_{x}^{0}$-coset, we may assume that $g T_{x} g^{-1}=T_{x}$, i.e. $g$ normalizes $T_{x}$. It follows that we obtain an exact sequence

$$
1 \rightarrow W_{x}^{0} \rightarrow W_{x} \rightarrow G_{x} / G_{x}^{0} \rightarrow 1
$$

of groups. Therefore,

$$
\begin{aligned}
\left(H^{*}\left(B T_{x}\right) \otimes I H_{x}^{*}(X ; \mathcal{L})\right)^{W_{x}} \cong\left(H^{*}\left(B T_{x} ; \mathcal{H}^{*}\left(R i_{x}^{!} I C^{T_{x}}(X ; \mathcal{L})\right)\right)^{W_{x}^{0}}\right)^{G_{x} / G_{x}^{0}} & \\
& \cong\left(H ^ { * } \left(B G_{x}^{0} ; \mathcal{H}^{*}\left(R i_{x}^{!} I C^{G_{x}^{0}}(X ; \mathcal{L})\right)^{G_{x} / G_{x}^{0}}\right.\right.
\end{aligned}
$$

where the first isomorphism follows from the short exact sequence above, and the second one from Theorem 1 (ii) with $G=N_{G_{x}^{0}}\left(T_{x}\right), H=C_{G_{x}^{0}}\left(T_{x}\right), X=x$ and $K=\mathcal{H}^{*}\left(R i_{x}^{!} I C^{N_{G_{x}^{0}}\left(T_{x}\right)}(X ; \mathcal{L})\right)$; one also needs to use the isomorphism

$$
H^{*}\left(B G_{x}^{0} ; \mathcal{H}^{*}\left(R i_{x}^{!} I C^{G_{x}^{0}}(X ; \mathcal{L})\right)\right) \cong H^{*}\left(B N_{G_{x}^{0}}\left(T_{x}\right) ; \mathcal{H}^{*}\left(R i_{x}^{!} I C^{N_{G_{x}^{0}}\left(T_{x}\right)}(X ; \mathcal{L})\right)\right.
$$

a consequence of (1.5.1). Finally, since $B G_{x}^{0}$ is simply connected, we have an isomorphism

$$
H^{*}\left(B G_{x}^{0} ; \mathcal{H}^{*}\left(R i_{x}^{!} I C^{G_{x}^{0}}(X ; \mathcal{L})\right)\right) \cong H^{*}\left(B G_{x}^{0}\right) \otimes I H_{x}^{*}(X ; \mathcal{L})
$$

And this isomorphism is equivariant for $G_{x} / G_{x}^{0}$ by Lemma (3.6).

Proof of Corollary 3. The first assertion is clear. Now the second assertion is also clear since $H^{*}\left(B G_{x}^{0}\right)$ is trivial in odd degrees. To obtain the third assertion one proceeds as follows. One may identify $D_{b}^{G_{x}}(x)$ with $D_{b}^{T_{x} \times F_{x}}(x)$ and one may replace the sheaves $\mathcal{H}^{*}\left(R i_{x}^{!} I C^{G_{x}}(X ; \mathcal{L})\right)$ with $\mathcal{H}^{*}\left(R i_{x}^{!} I C^{T_{x} \times F_{x}}(X ; \mathcal{L})\right)$. Now Theorem 1 (iii) with $X$ replaced by the point $x, K$ by $\mathcal{H}^{*}\left(R i_{x}^{!} I C^{T_{x} \times F_{x}}(X ; \mathcal{L})\right)$ and $G$ by $T_{x} \times F_{x}$ applies to complete the proof of Corollary 3.

\section{Spherical varieties and orbit closures in flag manifolds}

(4.1) A $G$-variety $X$ is spherical if $G$ is a connected reductive group and $X$ contains a dense orbit of a Borel subgroup $B$ of $G$. (Usually spherical varieties are assumed to be normal but we do not require this; see Lemma (4.2) below.) For $G$ a torus, we see that any toric variety is spherical.

Given a spherical variety $X$, let $\pi: \tilde{X} \rightarrow X$ denote the normalization. Then the $G$-action on $X$ extends to an action on $\tilde{X}$ making it a spherical variety and making the map $\pi$ equivariant. Recall that any spherical variety contains only finitely many $G$-orbits; as a consequence, it contains only finitely many fixed points of a maximal torus $T$ of $G$.

(4.2) Lemma. Let $X$ denote a spherical variety and let $\mathcal{L}$ denote a local system on the open $G$ orbit. Let $\pi: \tilde{X} \rightarrow X$ denote the normalization and let $\pi^{*}(\mathcal{L})=\tilde{\mathcal{L}}$. Let $I C(X ; \mathcal{L})(I C(\tilde{X} ; \tilde{\mathcal{L}}))$ denote the intersection cohomology complex of $X$ with respect to $\mathcal{L}$ (of $\tilde{X}$ with respect to $\tilde{\mathcal{L}}$, respectively ). Then the intersection cohomology sheaves $\mathcal{H}^{n}(I C(X ; \mathcal{L}))$ vanish for all odd $n$ if and only if the sheaves $\mathcal{H}^{n}(I C(\tilde{X} ; \tilde{\mathcal{L}}))$ vanish for all odd $n$.

Proof. Recall from $(1.7 .2)$ (i) that $R \pi_{*}(I C(\tilde{X} ; \tilde{\mathcal{L}})) \cong I C(X ; \mathcal{L})$. Now consider the Leray spectral sequence:

$$
\left.E_{2}^{s, t}=R^{s} \pi_{*} \mathcal{H}^{t}(I C(\tilde{X} ; \tilde{\mathcal{L}}))\right) \Rightarrow R^{s+t} \pi_{*}(I C(\tilde{X} ; \tilde{\mathcal{L}})) \cong \mathcal{H}^{s+t}\left(R \pi_{*}(I C(\tilde{X} ; \tilde{\mathcal{L}}))\right) \cong \mathcal{H}^{s+t}(I C(X ; \mathcal{L})) .
$$

Since $\pi$ is a finite map, $E_{2}^{s, t}=0$ for all $s>0$ in this spectral sequence; therefore one obtains the isomorphism $\pi_{*} \mathcal{H}^{t}(I C(\tilde{X} ; \tilde{\mathcal{L}})) \cong E_{2}^{0, t} \cong E_{\infty}^{0, t}=\mathcal{H}^{t}(I C(X ; \tilde{\mathcal{L}}))$. Now the lemma follows readily. 
(4.3.1) Next we review the local structure of spherical varieties. By the above lemma we may assume $X$ is a normal spherical variety. Now we recall the following from [Br-1] p. 399. Let $x \epsilon X$; choose a Borel subgroup $B$ of $G$ such that the orbit $B x$ is open in $G x$. Set

$$
X_{B x}=\{y \in X \mid \overline{B y} \supseteq B x\}
$$

Then $X_{B x}$ is affine, $B$-stable and Zariski open in $X$, and $X_{B x} \cap G x$ equals $B x$. Set

$$
P=\left\{g \in G \mid g X_{B x}=X_{B x}\right\}
$$

Then $P$ is a parabolic subgroup of $G$ containing $B$; let $R_{u}(P)$ denote its unipotent radical. Moreover, there exists a Levi subgroup $L$ of $P$, and an $L$-invariant closed subvariety $\Sigma$ of $X_{B x}$ containing $x$, such that

(4.3.4) the map $R_{u}(P) \times \Sigma \rightarrow X_{B x},(g, x) \rightarrow g . x$ is an isomorphism.

Then $\Sigma$ is an affine spherical $L$-variety that meets $G x$ along $L x$. Finally, the isotropy group $L_{x}$ contains the derived subgroup of $L$; in particular, $L_{x}$ is reductive. Thus, we can write

$$
\Sigma=L \times^{L_{x}} \mathcal{S}
$$

where $\mathcal{S}$ is a $L_{x}$-spherical affine variety containing $x$ as the unique fixed point, and $L \times{ }^{L_{x}} \mathcal{S}$ denotes the quotient of $L \times \mathcal{S}$ by the diagonal $L_{x}$-action. Then the natural map $L \times{ }^{L_{x}} \mathcal{S} \rightarrow L / L_{x}=L x$ is a fibration with fiber $\mathcal{S}$. It follows that $\mathcal{S}$ is a slice to $G x$ at $x$ for the $B$-action on $X$. Because $X$ is normal, $\Sigma$ and $\mathcal{S}$ are normal as well.

(4.4) Next we recall that any spherical $G$-variety $X$ admits an equivariant resolution of singularities, i.e., there exists a smooth $G$-variety $\tilde{X}$ together with a proper birational $G$-equivariant morphism $\pi$ : $\tilde{X} \rightarrow X$. Then the $G$-variety $\tilde{X}$ is also spherical; if moreover $X$ is complete, we may arrange so that $\tilde{X}$ is projective.

(4.5) Proposition. If $X$ is a complete spherical variety and $\mathcal{L}$ is a $G$-equivariant local system on the open $G$-orbit of $X$, then $I H^{n}(X ; \mathcal{L})=0$ for all odd $n$.

Proof. We will first use (1.7.2) (ii) to reduce to the case of the constant local system. We may first assume that $X$ is normal by (4.2). Next let $G x_{o} \cong G / G_{x_{o}}$ denote the open $G$-orbit in $X$. Let $\tilde{X}$ denote the normalization of $X$ in the function field $k\left(G / G_{x_{o}}^{0}\right)$. By $(1.7 .2)($ ii)

$$
I H^{n}(\tilde{X})=\underset{\chi}{\oplus} \operatorname{dim}(\chi) I H^{n}\left(X, \mathcal{L}_{\chi}\right)
$$

for all $n$, where the sum on the right hand side is over all the irreducible characters of the finite group $G_{x} / G_{x}^{0}$. Thus, it suffices to consider $X$ with the constant local system.

Next, let $\pi: \tilde{X} \rightarrow X$ denote a $G$-equivariant resolution of singularities, where $\tilde{X}$ is projective. Then, since $T$ acts on $\tilde{X}$ with only finitely many fixed points, we have $H^{n}(\tilde{X})=0$ for all odd $n$. (This follows e.g. from Theorem (1.5.3) since the the left-hand-side of $\left(1.5 .3 .^{*}\right)$ is now $H^{*}(B T)_{(0)} \otimes H^{*}(\tilde{X})$.) Now the decomposition theorem in intersection cohomology shows that $I H^{n}(X)$ is a summand of $H^{n}(\tilde{X})$ for any $n$. The latter is trivial for all odd $n$; this completes the proof of the Proposition.

(4.6) Lemma. Let $X$ be a spherical variety, $\mathcal{L}$ be a $G$-equivariant local system on the open $G$-orbit and let $x \in X$ be a fixed point of a maximal torus $T$. Then the stalks at $x$ of the cohomology sheaves $\mathcal{H}^{n}(I C(X ; \mathcal{L}))$ vanish for all odd $n$.

Proof. By Lemma 4.2, we may assume that $X$ is normal. Then $x$ admits an open $G$-stable quasiprojective neighborhood $U_{x}$ (see $[\mathrm{Su}]$ ). Thus, we may replace $X$ by the closure of $U_{x}$, and assume that $X$ is projective. Now we conclude by Theorem (1.5.3) applied to $\mathcal{L}^{\vee}$ in the place of $\mathcal{L}$, together with Proposition (4.5). (Observe that the fundamental group of the open orbit, $\pi_{1}\left(G x_{o}\right)$ acts on the stalks of 
$\mathcal{L}$ through its image in $\pi_{1}\left(E G \underset{G}{\times} G x_{o}\right)$ which is finite. Therefore $\mathcal{L}$ is semi-simple as a local system, and Theorem (1.5.3) applies.)

(4.7) Proof of Theorem 4. We argue by induction on the dimension of $X$. So we may assume that the theorem is true for all spherical varieties of strictly smaller dimension. Moreover, in view of (4.2), we may assume that $X$ is normal. Now, if $x$ is fixed by $G$, then $\mathcal{H}^{n}(I C(X ; \mathcal{L}))_{x}=0$ for all odd $n$, by Lemma (4.6).

The $G$-equivariant local system $\mathcal{L}$ restricts to a $P$-equivariant local system on a $P$-stable open subvariety of $X_{B x}$. Under the isomorphisms in (4.3.4) and (4.3.5), this corresponds to an $L_{x}$-equivariant local system $\mathcal{L}_{\mathcal{S}}$ on the open $L_{x}$-orbit on $\mathcal{S}$. (Observe that the isomorphism in (4.3.4) is $L$-equivariant, when $L$ acts on the left hand side by $l(u, s)=\left(l u l^{-1}, l s\right), l \epsilon L, u \in R_{u}(P)$ and $s \epsilon \Sigma$.) If $x$ is not fixed by $G$, then $\operatorname{dim}(\mathcal{S})<\operatorname{dim}(X)$ and (4.3.4), (4.3.5) imply that

$$
\mathcal{H}^{n}(I C(X ; \mathcal{L}))_{x} \cong \mathcal{H}^{n}\left(I C\left(\mathcal{S} ; \mathcal{L}_{\mathcal{S}}\right)\right)_{x}
$$

for all $n$. Now we conclude by the inductive hypothesis in this case.

In the rest of this section we consider the orbit closures of symmetric subgroups in flag manifolds.

(4.8) Let $G$ denote a connected reductive group and let $\theta$ be an automorphism of order two of the algebraic group $G$. Let $K$ denote the fixed point subgroup of $\theta$ and let $B$ denote a Borel subgroup of $G$. Now $K$ acts on the flag manifold $G / B$. It follows from [Sp] and [M-S] that the hypotheses of Theorem 2 are satisfied by the closures of the $K$-orbits on $G / B$. The main result of this section is that the global odd dimensional intersection cohomology groups of these orbit closures with respect to the constant local system are trivial. Now one may apply Theorem 3 (ii) which provides the vanishing of an invariant part of odd dimensional intersection cohomology sheaves.

(4.9) First recall that $K$ need not be connected in general; by a theorem of Steinberg, $K$ is connected if $G$ is semi-simple and simply connected (see [St].) The following are now standard examples of the above situation.

Examples (See [R-S] section 10.)

(4.9.1) Let $\mathbf{G}$ denote a connected reductive group and $\mathbf{B}$ a Borel subgroup of $\mathbf{G}$. Now let $G=\mathbf{G} \times \mathbf{G}$ with the involution $\theta$ interchanging the two factors. Then $K=\mathbf{G}$ and, by the Bruhat decomposition, the $K$-orbits on $\mathbf{G} / \mathbf{B} \times \mathbf{G} / \mathbf{B}$ are parameterized by the Weyl group of $G$. In this case each stratum has a fixed point for the action of a maximal torus of $\mathbf{G}$ and therefore the theorem in (1.5.3) applies.

(4.9.2) Let $G=G L_{n}$ and let $\theta$ be defined by $\theta(g)={ }^{t} g^{-1}$. Now the fixed point group of $\theta$ is the orthogonal group $O_{n}$. We may also let $G=P G L_{n}$ and let $\theta$ on $P G L_{n}$ be induced by the involution $\theta$ on $G L_{n}$.

(4.9.3) Let $G=S L_{n}$ and let $\theta$ be defined by $\theta(g)={ }^{t} g^{-1}$. Now the fixed point group of $\theta$ is the special orthogonal group $\mathrm{SO}_{n}$.

(4.9.4) Let $G=S L_{2 n}$ and let $J \epsilon G$ be defined by $J\left(\mathbf{e}_{i}\right)=-\mathbf{e}_{n+i}$ and $J\left(\mathbf{e}_{n+i}\right)=\mathbf{e}_{i}, i=1, \ldots, n$ where $\mathbf{e}_{1}, \ldots, \mathbf{e}_{2 n}$ is the standard basis of $k^{2 n}$. Define $\theta$ by $\theta(g)=J\left({ }^{t} g\right)^{-1} J^{-1}$. Now the fixed point group $K$ is the symplectic group $S p_{2 n}$.

The following result is a consequence of [R-S] Theorem 4.6.

(4.10) Proposition. Let $\overline{\mathcal{O}}$ denote the closure of a $K$-orbit in $G / B$. If $\mathcal{O}$ is not closed, then there exists a minimal parabolic subgroup $P \supset B(P \neq B)$ and a $K$-orbit closure $\overline{\mathcal{O}}^{\prime} \subset \overline{\mathcal{O}}$ such that

(i) $\overline{\mathcal{O}}=\pi^{-1}\left(\pi\left(\overline{\mathcal{O}}^{\prime}\right)\right)$ and

(ii) the map $\pi^{\prime}=\pi \mid \overline{\mathcal{O}}^{\prime}: \overline{\mathcal{O}}^{\prime} \rightarrow \pi\left(\overline{\mathcal{O}}^{\prime}\right)$ is generically finite, where $\pi: G / B \rightarrow G / P$ is the projection. Then $\pi \mid \overline{\mathcal{O}}: \overline{\mathcal{O}} \rightarrow \pi\left(\overline{\mathcal{O}}^{\prime}\right)$ is a projective line bundle. 
(4.11) Theorem. Let $\overline{\mathcal{O}}$ denote the closure of a $K$-orbit in $G / B$. Now $I H^{n}(\overline{\mathcal{O}})=0$ for all odd $n$.

Proof. We will prove this theorem using ascending induction on the dimension of the orbit $\mathcal{O}$, so that the induction starts with a closed orbit $\mathcal{O}$. Identify $\mathcal{O}$ with $K /\left(K \cap g B g^{-1}\right)$ for some $g \in G$. Because $\mathcal{O}$ is projective and $B$ is solvable, $\left(K \cap g B g^{-1}\right)^{0}$ is a Borel subgroup of $K^{0}$. Thus, $\mathcal{O}$ is a finite union of disjoint copies of the flag manifold of $K^{0}$; therefore, $\left.I H^{n}(\mathcal{O})\right) \cong H^{n}(\mathcal{O})$ vanishes for all odd $n$.

Next consider a $K$-orbit closure $\overline{\mathcal{O}}$ in $G / B$ such that for all orbit closures $\overline{\mathcal{O}^{\prime}}$ properly contained in $\overline{\mathcal{O}}, I H^{n}\left(\overline{\mathcal{O}}^{\prime}\right)=0$ if $n$ is odd. Now choose an orbit closure $\overline{\mathcal{O}}^{\prime}$ as in $(4.10)$. Observe that $\overline{\pi(\mathcal{O})}=\pi(\overline{\mathcal{O}})=$ $\pi\left(\overline{\mathcal{O}}^{\prime}\right)=\overline{\pi\left(\mathcal{O}^{\prime}\right)}$ and therefore $\pi(\mathcal{O})=\pi\left(\mathcal{O}^{\prime}\right)$

Consider the proper, generically finite map $\pi^{\prime}: \overline{\mathcal{O}}^{\prime} \rightarrow \pi\left(\overline{\mathcal{O}}^{\prime}\right)$. Now $\pi^{\prime-1} \pi^{\prime}\left(\mathcal{O}^{\prime}\right)=\mathcal{O}^{\prime}$ and $\pi_{*}^{\prime} \pi^{\prime *}(\underline{\mathbb{Q}})$ is a $K$-equivariant local system on $\pi\left(\mathcal{O}^{\prime}\right)$. Let $I C\left(\overline{\mathcal{O}}^{\prime}\right)\left(I C\left(\pi\left(\overline{\mathcal{O}}^{\prime}\right) ; \pi^{\prime}{ }_{*} \pi^{\prime *}(\underline{\mathbb{Q}})\right)\right)$ denote the intersection cohomology complex on $\overline{\mathcal{O}}^{\prime}$ obtained by starting with $\mathbb{Q}$ on $\mathcal{O}^{\prime}$ (on $\pi\left(\overline{\mathcal{O}}^{\prime}\right)$ obtained by starting with $\pi_{*}^{\prime} \pi^{\prime *} \mathbb{Q}$ on $\pi\left(\mathcal{O}^{\prime}\right)$, respectively.) The decomposition theorem in intersection cohomology shows that

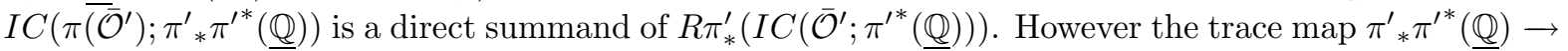
$\underline{\mathbb{Q}}$ shows that $I C\left(\bar{\pi}\left(\overline{\mathcal{O}}^{\prime}\right)\right)$ is a direct summand of $I C\left(\pi\left(\overline{\mathcal{O}^{\prime}}\right) ;{\overline{\pi^{\prime}}}^{\prime} \pi^{\prime *}(\underline{\mathbb{Q}})\right)$. It follows that $I C\left(\pi\left(\overline{\mathcal{O}}^{\prime}\right)\right)$ is a direct summand of $R \pi^{\prime}{ }_{*} I C\left(\overline{\mathcal{O}}^{\prime} ; \pi^{\prime *}(\underline{\mathbb{Q}})\right)$, whence

$I H^{n}\left(\pi\left(\overline{\mathcal{O}}^{\prime}\right)\right)$ is a direct summand of $I H^{n}\left(\overline{\mathcal{O}}^{\prime}\right)$ for all $n$.

Since $I H^{n}\left(\overline{\mathcal{O}}^{\prime}\right)=0$ for all odd $n$, it follows that $I H^{n}\left(\pi\left(\overline{\mathcal{O}}^{\prime}\right)\right)=0$ for all odd $n$ as well.

Next recall from $(4.10)$ that $\overline{\mathcal{O}}=\pi^{-1}\left(\pi\left(\overline{\mathcal{O}}^{\prime}\right)\right)$ is a projective line bundle over $\pi\left(\overline{\mathcal{O}}^{\prime}\right)$. Therefore it follows that $I H^{n}(\overline{\mathcal{O}})=0$ for all odd $n$ as well.

To complete the proof of Theorem 5, we now apply Theorem 2 to the closure of a $K$-orbit in $G / B$. Then the hypothesis on the existence of attractive slices is satisfied by [M-S] (6.4). Moreover, each stabilizer is the semi-direct product of a connected solvable group and a finite group, as shown in [Sp] (4.8). Therefore Corollary 3 completes the proof of Theorem 5. 


\section{Appendix.}

Now we return to the setting of (0.3) and (0.4).

(A.1) Lemma. For a torus $T$ acting on a variety $X$ with a fixed point $x$, the following conditions are equivalent:

(i) $x$ is attractive, that is, there exists a one parameter subgroup $\lambda: \mathbb{G}_{m} \rightarrow T$ such that, for any $y$ in a Zariski open neighborhood of $x$, we have $\lim _{t \rightarrow 0} \lambda(t) y=x$.

(ii) The weights of $T$ in the Zariski tangent space $\mathcal{T}_{x} X$ are contained in an open half space.

Moreover, if (i) or (ii) holds, then $x$ admits a unique open affine $T$-stable neighborhood in $X$, and the latter admits a closed equivariant embedding into $\mathcal{T}_{x} X$.

Proof. Because $X$ is locally linear, $x$ admits an open affine $T$-stable neighborhood in $X$. To prove the equivalence of (i) and (ii), we may replace $X$ by this neighborhood, and assume that $X$ is affine. Then $X$ admits a closed equivariant embedding into a $T$-module $V$.

Now we will prove that (i) implies (ii). Indeed, (i) implies that $X$ is contained in the sum of all positive eigen spaces of $V$ (for the induced action of $\mathbb{G}_{m}$ ). Thus, $\mathbb{G}_{m}$ acts with negative weights on the maximal ideal of $X$ at $x$, and (ii) follows.

Next we will prove (ii) implies (i). Let $A$ be the algebra of regular functions on $X$ and let $m$ be the maximal ideal of $A$ associated with $x$. Then there exists a one parameter subgroup $\lambda$ such that all weights of $\mathbb{G}_{m}$ in $m / m^{2}$ (the dual of $\mathcal{T}_{x} X$ ) are negative when evaluated on $\lambda$. Let $g r m$ be the direct sum of the $m^{n} / m^{n+1}$ over all positive $n$. Then the weights of $g r m$ are negative, too. But $m$ is isomorphic to gr $m$ as a $T$-module, because $T$ is linearly reductive. It follows that $\lim _{t \rightarrow 0} f(\lambda(t) y)=0$ for all $f \in m$ and $y \in X$. This implies (i).

Now we consider the last assertion: Let $X_{x}$ be the set of all $y \in X$ such that $\lim _{t \rightarrow 0} \lambda(t) y=x$. Then $X_{x}$ is contained in any open $T$-stable neighborhood of $x$ in $X$. If moreover $U_{x}$ is an affine $T$-stable neighborhood, then $X_{x}=U_{x}$ (otherwise $U_{x}-X_{x}$ is closed and $T$-stable, hence contains a closed $T$-orbit; but this is absurd because all $T$-invariant regular functions on $U_{x}$ are constant.)

So we may assume that $X=X_{x}$. Then the algebra $A$ of regular functions on $X$ is negatively graded, by the action of $\lambda$. Choose a $T$-stable complement $M$ to $m^{2}$ in $m$. Then $M$ generates the algebra $A$ (by the graded version of Nakayama's lemma). Thus, the corresponding morphism $X \rightarrow M^{*}$ is a closed immersion. This morphism is $T$-equivariant, and $M$ is isomorphic to $\mathrm{m} / \mathrm{m}^{2}$ whence $M^{*}$ is isomorphic to $\mathcal{T}_{x} X$.

(A.2) Let $\mathbb{G}_{m}$ act linearly on an affine space $\mathbb{A}^{n}$ with positive weights. Now the geometric quotient $\left(\mathbb{A}^{n}-0\right) / \mathbb{G}_{m}$ is a weighted projective space which is a rationally smooth variety (see [Do] (2.3.6) for example). We will denote this by $\mathbb{P}$. The quotient map $\pi^{\prime}: \mathbb{A}^{n}-0 \rightarrow \mathbb{P}$ has fibers all isomorphic to $\mathbb{G}_{m}$. Moreover one obtains a commutative square

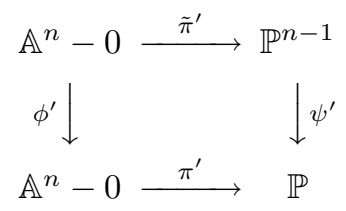

where the maps $\phi^{\prime}$ and $\psi^{\prime}$ denote taking the quotients with respect to a suitable finite group.

Now let $Y$ denote a closed sub-variety of $\mathbb{P}$ and let $\tilde{Y}=\underset{\mathbb{P}}{n-1} \underset{\mathbb{P}}{\times Y}, S^{\prime}=\left(\mathbb{A}^{n}-0\right) \underset{\mathbb{P}}{\times}$ and $\tilde{S}^{\prime}=$ $\left(\mathbb{A}^{n}-0\right) \underset{\mathbb{P}}{\times S}$ denote their inverse images by the maps $\psi^{\prime}, \pi^{\prime}$ and the composition $\pi^{\prime} \circ \phi^{\prime}$. Let $S=\bar{S}^{\prime}$ $\left(\tilde{S}=\overline{\tilde{S}}^{\prime}\right)$ denote the closure of $S^{\prime}\left(\tilde{S}^{\prime}\right.$, respectively $)$. We now obtain another commutative square:

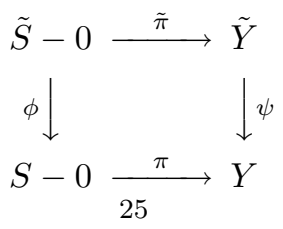


(A.3) Proposition. Assume the above situation.

(i) Now $R^{n} \pi_{!}(\underline{\mathbb{Q}})=\underline{\mathbb{Q}}$ if $n=1,2$ and $R^{n} \pi_{*}(\underline{\mathbb{Q}})=\underline{\mathbb{Q}}$ if $n=0,1$.

(ii) Let $\mathcal{L}$ denote a local system on an open smooth subvariety of $Y$ and let $\mathcal{L}_{S}$ denote the pull-back of $\mathcal{L}$ to an open smooth subvariety $S_{0}$ of $S-0$. Let $I C(Y, \mathcal{L})$ and $I C\left(S-0, \mathcal{L}_{S}\right)$ denote the corresponding intersection cohomology complexes. Then

$$
\begin{aligned}
& \mathcal{H}_{\text {perv }}^{n}\left(R \pi_{!}\left(I C\left(S-0 ; \mathcal{L}_{S}\right)\right)\right)=I C(Y ; \mathcal{L}) \text { if } n=1,2 \text { and } \\
& \mathcal{H}_{\text {perv }}^{n}\left(R \pi_{*}\left(I C\left(S-0 ; \mathcal{L}_{S}\right)\right)\right)=I C(Y ; \mathcal{L}) \text { if } n=0,1
\end{aligned}
$$

where $\mathcal{H}_{\text {perv }}^{n}$ denotes the perverse cohomology objects (computed using the perverse $t$-structure on $Y$ ).

Proof. The first statement is clear since the fibers of the map $\pi$ are isomorphic to $\mathbb{G}_{m}$. Now we consider the second statement. Let $\mathcal{L}$ be defined on the open subvariety $Y_{0}$ of $Y$. Let $\tilde{\mathcal{L}}\left(\tilde{\mathcal{L}}_{\tilde{S}}\right)$ denote the pull-back of $\mathcal{L}$ to an open smooth subvariety $\tilde{Y}_{0}$ of $\tilde{Y}\left(\tilde{S}_{0}\right.$ of $\tilde{S}-0$, respectively ). (We may assume that $Y_{0}$ is the quotient of $\tilde{Y}_{0}$ and $S_{0}$ is the quotient of $\tilde{S}_{0}$ by the finite groups in (A.2.1).) We will denote by $\psi_{0}: \tilde{Y}_{0} \rightarrow Y_{0}$ and $\phi_{0}: \tilde{S}_{0} \rightarrow S_{0}$ the corresponding finite maps.

Let $I C(\tilde{Y} ; \tilde{\mathcal{L}})$ and $I C\left(\tilde{S}-0 ; \tilde{\mathcal{L}}_{\tilde{S}}\right)$, denote the corresponding intersection cohomology complexes. Now observe that the map $\tilde{\pi}$ is smooth. Therefore

$$
\tilde{\pi}^{*} I C(\tilde{Y} ; \tilde{\mathcal{L}})=I C\left(\tilde{S}-0 ; \tilde{\mathcal{L}}_{\tilde{S}}\right)
$$

Now we apply $R \pi_{!} \circ \phi_{*}=R \pi_{!} \circ R \phi_{!}=R \psi_{!} \circ R \tilde{\pi}_{!}=\psi_{*} \circ R \tilde{\pi}_{!}$to both sides of (A.3.1). By the projection formula:

$$
R \tilde{\pi}_{!}\left(I C\left(\tilde{S}-0 ; \tilde{\mathcal{L}}_{\tilde{S}}\right)\right)=I C(\tilde{Y} ; \tilde{\mathcal{L}}) \underset{\mathbb{Q}}{\otimes} R \pi !(\underline{\mathbb{Q}})
$$

The distinguished triangle

$$
I C(\tilde{Y} ; \tilde{\mathcal{L}}) \underset{\mathbb{Q}}{\otimes} \tau_{\leq 1} R \pi !(\underline{\mathbb{Q}}) \rightarrow I C(\tilde{Y} ; \tilde{\mathcal{L}}) \underset{\mathbb{Q}}{\otimes} R \pi !(\underline{\mathbb{Q}}) \rightarrow I C(\tilde{Y} ; \tilde{\mathcal{L}}) \underset{\mathbb{Q}}{\otimes \tau_{\geq 2}} R \pi !(\underline{\mathbb{Q}})
$$

provides us with a long exact sequence in perverse cohomology using which one readily computes

$(\mathrm{A} .3 .3) \mathcal{H}_{\text {perv }}^{n}\left(R \tilde{\pi}_{!}\left(I C\left(\tilde{S}-0 ; \tilde{\mathcal{L}}_{\tilde{S}}\right)\right)\right)=I C(\tilde{Y} ; \tilde{\mathcal{L}})$ if $n=1,2$ and is trivial otherwise.

Observe that $\psi_{*}$ and $\phi_{*}$ are $t$-exact, and therefore preserve perverse objects. Therefore

(A.3.4) $\mathcal{H}_{\text {perv }}^{n}\left(R \pi ! \phi_{*}\left(I C\left(\tilde{S}-0 ; \tilde{\mathcal{L}}_{\tilde{S}}\right)\right)\right)=\mathcal{H}_{\text {perv }}^{n}\left(\psi_{*} R \tilde{\pi}_{!}\left(I C\left(\tilde{S}-0 ; \tilde{\mathcal{L}}_{\tilde{S}}\right)\right)\right)$

$=\psi_{*}(I C(\tilde{Y} ; \tilde{\mathcal{L}}))=I C\left(Y ; \psi_{0 *}(\tilde{\mathcal{L}})\right)$ if $n=1,2$ and is trivial otherwise.

Now the local system $\psi_{0 *}(\tilde{\mathcal{L}})=\psi_{0 *}\left(\psi_{0}^{*}(\mathcal{L})\right)$ equals $\mathcal{L} \oplus \mathcal{L}^{\prime}$ for some other local system $\mathcal{L}^{\prime}$. This follows from the observation that the composition $\mathcal{L} \rightarrow \psi_{0 *}\left(\psi_{0}^{*}(\mathcal{L})\right) \rightarrow \mathcal{L}$ is the identity. Similarly, $\phi_{0 *}\left(\tilde{\mathcal{L}}_{\tilde{S}}\right)=\phi_{0 *}\left(\phi_{0}^{*}\left(\mathcal{L}_{S}\right)\right)=\mathcal{L}_{S} \oplus \mathcal{L}_{S}^{\prime \prime}$ where $\mathcal{L}_{S}^{\prime \prime}$ is another local system on $S_{0}$. Therefore

$$
\begin{aligned}
& \phi_{*}\left(I C\left(\tilde{S}-0 ; \tilde{\mathcal{L}}_{\tilde{S}}\right)\right)=I C\left(S-0 ; \phi_{0 *}\left(\tilde{\mathcal{L}}_{\tilde{S}}\right)\right)=I C\left(S-0 ; \mathcal{L}_{S}\right) \oplus I C\left(S ; \mathcal{L}_{S}^{\prime \prime}\right) \text { and } \\
& I C\left(Y ; \psi_{0 *}(\tilde{\mathcal{L}})\right)=I C(Y ; \mathcal{L}) \oplus I C\left(Y ; \mathcal{L}^{\prime}\right) .
\end{aligned}
$$

Now the left hand-side of (A.3.4) becomes $\mathcal{H}_{\text {perv }}^{n}\left(R \pi_{!} I C\left(S-0 ; \mathcal{L}_{S}\right)\right) \oplus \mathcal{H}_{\text {perv }}^{n}\left(R \pi_{!} I C\left(S-0 ; \mathcal{L}_{S}^{\prime \prime}\right)\right)$ while the right hand side of $(\mathrm{A} .3 .4)$ becomes $I C(Y ; \mathcal{L}) \oplus I C\left(Y ; \mathcal{L}^{\prime}\right)$ for $n=1,2$. Since the perverse $t$-structure on the smooth stratum is the usual $t$-structure and the projection formula shows that $R \pi_{!}\left(I C\left(S-0 ; \mathcal{L}_{S}\right)\right)$ agrees with $I C(Y ; \mathcal{L}) \otimes R \pi !(\underline{\mathbb{Q}})$ on the smooth stratum, we may conclude that

$$
\mathcal{H}_{\text {perv }}^{n}\left(R \pi_{!} I C\left(S-0 ; \mathcal{L}_{S}\right)\right)=I C(Y ; \mathcal{L}) \text { for } n=1,2 \text { and is trivial otherwise. }
$$

This proves the second statement for $R \pi_{!}$. The second statement for $R \pi_{*}$ now follows by taking Verdier duals. 


\section{References}

[A-K] A. B. Altman, and S. L. Kleiman, Introduction to Grothendieck duality theory, Lecture Notes in Math. 146, Springer-Verlag, 1970

[B-B-F-K] G. Barthel, J-P. Brasselet, K-H. Fieseler and L. Kaup: Equivariant intersection cohomology of toric varieties, in: Algebraic geometry: Hirzebruch 70 (Warsaw, 1998), Contemp. Math., 241, AMS, Providence (1999)

[B-B-D] J. Bernstein, A. Beilinson, and P. Deligne, Faisceaux pervers, Astérisque 100, (1981)

[B-L] J. Bernstein, and V. Lunts, Equivariant sheaves and functors, Lecture Note in Math. 1578, Springer-Verlag, 1994

[Br-1] M. Brion, Groupe de Picard et nombres caractéristiques des variétés sphériques, Duke Math. Journal, 58, no. 2, (1989), 397-424

[Br-2] M. Brion, Rational smoothness and fixed points of torus actions, Transformation Groups 4 (1999), 127-156

[Bryl] J-L. Brylinski, Equivariant intersection cohomology, in: Kazhdan-Lusztig theory and related topics, Contemp. Math., 139, AMS, Providence (1992)

[De] P. Deligne, Théorème de Lefschetz et critères de dégénerescence des suites spectrales, Publ. Math. IHES, 35, (1968), 259-278

[Do] I. Dolgachev, Weighted projective varieties, Lect. Notes in Math., 956, 35-71, SpringerVerlag, 1982

[Fr] E. Friedlander, Etale homotopy of simplicial schemes, Ann. Math. Studies 104, (1983)

[G-M] M. Goresky and R. Macpherson, Intersection Cohomology II, Invent. Math., 72, (1983) pp. 77- 129

[J-1] R. Joshua, Vanishing of odd dimensional intersection cohomology, Math. Z., 195, (1987), 239-253

[J-2] R. Joshua, The intersection cohomology and the derived category of algebraic stacks, in Algebraic K-theory and Algebraic Topology, NATO ASI Series C, 407, (1993), 91 -145. See also: http://www.math.ohio-state.edu/ joshua

[J-3] R. Joshua, Equivariant Riemann-Roch for G-quasi-projective varieties, K-theory, 17, no. 1, (1999), 1-35

[J-4] R. Joshua, Derived functors for maps of simplicial spaces, preprint (1999)

[J-5] R. Joshua, Vanishing of odd dimensional intersection cohomology for spherical varieties in positive characteristics, preprint (in preparation)

[K-L] D. A. Kazhdan, and G. Lusztig, Schubert varieties and Poincaré duality, Proc. Sympos. Pure Math., 36, (1979), 185-203

[K] F. Kirwan, Cohomology of quotients in symplectic and algebraic geometry, Princeton Lect. Notes, 31, (1984)

[K-M] I. Kritz, I and P. May, Operads, algebras, modules and motives, Astérisque, 233, (1996)

[L-V] G. Lusztig, and D. Vogan, Singularities of closures of $K$-orbits on flag manifolds, Invent. Math., 71, (1983), 365-379

[M-S] J.G.M. Mars, and T. A. Springer, Hecke algebra representation related to spherical varieties, Representation Theory, 2, 33-69, (1998)

[R-S] R. W. Richardson, and T. A. Springer, The Bruhat order on symmetric varieties, Geometriae Dedicata, 35, (1990), 389-436 
[Sp] T. A. Springer, Algebraic groups with involutions, Advanced Studies in Pure Math., 6: Algebraic groups and related topics, (1985), 525-543

[St] R. Steinberg, Endomorphisms of linear algebraic groups, Mem. Amer. Math. Soc., 80, (1968)

[Su] H. Sumihiro, Equivariant completion, J. Math. Kyoto Univ., 14, (1974), 1-28

Institut Fourier, BP 74

38402 Saint-Martin d'Hères,

France.
Department of Mathematics,

The Ohio State University,

Columbus, Ohio-43210, USA 